J. Jpn. Soc. Comp. Statist., 22(2009), 1-32

\title{
LONGITUDINAL AND INCOMPLETE CLINICAL STUDIES
}

\author{
Geert Molenberghs $^{* \dagger}$ and Geert Verbeke ${ }^{\dagger *}$
}

\begin{abstract}
Repeated measures are obtained whenever an outcome is measured repeatedly within a set of units. The fact that observations from the same unit, in general, will not be independent poses particular challenges to the statistical procedures used for the analysis of such data. The current paper is dedicated to an overview of frequently used statistical models for the analysis of repeated measurements, with emphasis on model formulation and parameter interpretation.

Missing data frequently occur in repeated measures studies, especially in humans. An important source for missing data is patients who leave the study prematurely, so-called dropouts. When patients are evaluated only once under treatment, then the presence of dropouts makes it hard to comply with the intention-to-treat (ITT) principle. However, when repeated measurements are taken then one can make use of the observed portion of the data to retrieve information on dropouts. Generally, commonly used methods to analyze incomplete longitudinal clinical trial data include complete-case (CC) analysis and an analysis using the last observation carried forward (LOCF). However, these methods rest on strong and unverifiable assumptions about the dropout mechanism. Over the last decades, a number of longitudinal data analysis methods have been suggested, providing a valid estimate for, e.g., the treatment effect under less restrictive assumptions.

We will argue that direct likelihood methods, using all available data, require the relatively weak missing at random assumption only. Likewise, weighted generalized estimating equations and multiple imputation are discussed. Finally, because it is impossible to verify that the dropout mechanism is MAR, we argue that, to evaluate the robustness of the conclusion, a sensitivity analysis thereby varying the assumption on the dropout mechanism should become a standard procedure when analyzing the results of a clinical trial.
\end{abstract}

\section{Introduction}

In medical science, studies are often designed to investigate changes in a specific parameter which is measured repeatedly over time in the participating subjects. Such studies are called longitudinal studies, in contrast to cross-sectional studies where the response of interest is measured only once for each individual. As pointed out by Diggle et al. (2002) the main advantage of longitudinal studies is that they can distinguish changes over time within individuals (longitudinal effects) from differences among people in their baseline values (cross-sectional effects).

In randomized clinical trials, where the aim is usually to compare the effect of two (or more) treatments at a specific time-point, the need and the advantage of taking repeated

\footnotetext{
${ }^{*}$ I-BioStat, Universiteit Hasselt, Agoralaan, B3590 Diepenbeek, Belgium

${ }^{\dagger}$ I-BioStat, Katholieke Universiteit Leuven, Kapucijnenvoer 35, B3000 Leuven, Belgium

Key words: Longitudinal data; Inverse probability weighting; Marginal models; Missing at random, Mixed models; Multiple imputation.
} 
measures is at first sight less obvious. Indeed, a simple comparison of the treatment groups at the end of the follow-up period is often sufficient to establish the treatment effect(s) (if any) by virtue of the randomization. However, in some instances, it is important to know how the patients have reached their endpoint, i.e., it is important to compare the average profiles (over time) between the treatment groups. Further, longitudinal studies can be more powerful than studies evaluating the treatments at one single time-point. Finally, follow-up studies often suffer from dropout, i.e., some patients leave the study prematurely, for known or unknown reasons. In such cases, a full repeated measures analysis will help in drawing inferences at the end of the study. Since incompleteness usually occurs for reasons outside of the control of the investigators and may be related to the outcome measurement of interest, it is generally necessary to reflect on the process governing incompleteness. Only in special but important cases is it possible to ignore the missingness process.

When patients are examined repeatedly in a clinical trial, missing data can occur for various reasons and at various visits. When missing data result from patient dropout, the missing data pattern is monotone pattern. Non-monotone missingness occurs when there are intermittent missing values as well. Our focus will be on dropout.

When referring to the missing-value, or non-response, process we will use the terminology of Little and Rubin (2002). A non-response process is said to be missing completely at random (MCAR) if the missingness is independent of both unobserved and observed data and missing at random (MAR) if, conditional on the observed data, the missingness is independent of the unobserved measurements. A process that is neither MCAR nor MAR is termed non-random (MNAR). In the context of likelihood inference, and when the parameters describing the measurement process are functionally independent of the parameters describing the missingness process, MCAR and MAR are ignorable, while a non-random process is non-ignorable. Thus, under ignorable dropout, one can literally ignore the missingness process and nevertheless obtain valid estimates of, say, the treatment effect. Above definitions are conditional on including the correct set of covariates into the model. An overview of the various mechanisms, and their (non-)ignorability under likelihood, Bayesian, or frequentist inference, is given in Table 1.

Let us first consider the case where one follow-up measurement per patient is made. When dropout occurs, and hence there are no follow-up measurements, one usually is forced to discard such a patient from analysis, thereby violating the intention to treat (ITT) principle which stipulates that all randomized patients should be included in the primary analysis and according to the randomization scheme. Of course, the effect of treatment can be investigated under extreme assumptions, such as, fore example, a worst case and a best case scenario, but such scenarios are most often not really helpful.

Early work regarding missingness focused on the consequences of the induced lack of balance of deviations from the study design (Afifi and Elashoff 1966; Hartley and Hocking 1971). Later, algorithmic developments took place, such as the expectation-maximization

Table 1: Overview of missing data mechanisms.

\begin{tabular}{llll}
\hline \hline Acronym & Description & Likelih./Bayesian & Frequentist \\
\hline MCAR & missing completely at random & ignorable & ignorable \\
MAR & missing at random & ignorable & non-ignorable \\
MNAR & missing not at random & non-ignorable & non-ignorable \\
\hline \hline
\end{tabular}


algorithm (EM; Dempster, Laird and Rubin, 1977) and multiple imputation (Rubin 1987). These have brought likelihood-based ignorable analysis within reach of a large class of designs and models. However, they usually require extra programming in addition to available standard statistical software.

In the meantime, however, clinical trial practice has put a strong emphasis on methods such as complete case analysis (CC) and last observation carried forward (LOCF) or other simple forms of imputation. Claimed advantages include computational simplicity, no need for a full longitudinal model analysis (e.g., when the scientific question is in terms of the last planned measurement occasion only) and, for LOCF, compatibility with the ITT principle. However, a CC analysis assumes MCAR and the LOCF analysis makes peculiar assumptions on the (unobserved) evolution of the response, underestimates the variability of the response and ignores the fact that imputed values are no real data.

On the other hand, a likelihood-based longitudinal analysis requires only MAR, uses all data (obviating the need for both deleting and filling in data) and is also consistent with the ITT principle. Further, it can be shown that also the incomplete sequences contribute to estimands of interest (treatment effect at the end of the study), even early dropouts. For continuous responses, the linear mixed model is quite popular and is a direct extension of ANOVA and MANOVA approaches, but more broadly valid in incomplete data settings. For categorical responses and count data, so-called marginal (e.g., generalized estimating equations, GEE) and random-effects (e.g., generalized linear mixed-effects models, GLMM) approaches are in use. While GLMM parameters can be fitted using maximum likelihood, the same is not true for the frequentist GEE method but modifications have been proposed to accommodate the MAR assumption (Robins, Rotnitzky and Zhao, 1995).

Finally, MNAR missingness can never be fully ruled out based on the observed data only. It is argued that, rather than going either for discarding MNAR models entirely or for placing full faith on them, a sensible compromise is to make them a component of a sensitivity analysis.

Case studies are introduced in Section 2. In Section 3, we will first focus on linear models for Gaussian data. In Section 4, we will discuss models for the analysis of discrete outcomes. Section 5. describes simple methods to deal with incomplete data, while more appropriate methods are described in Section 6. An illustration is provided in Section 7. A perspective on computation aspects is provided in Section 8. Sensitivity analysis is briefly discussed in Section 9.

\section{Case studies}

\subsection{The toenail data}

As a typical longitudinal example, we consider data from a randomized, double blind, parallel group, multicentre study for the comparison of two oral treatments (in the sequel coded as $A$ and $B$ ) for toenail dermatophyte onychomycosis (TDO). We refer to De Backer et al. (1996) for more details about this study. TDO is a common toenail infection, difficult to treat, affecting more than two percent of the population. Antifungal compounds classically used for treatment of TDO need to be taken until the whole nail has grown out healthy. However, new compounds, have reduced the treatment duration to three months. The aim of the present study was to compare the efficacy and safety of two such new compounds, labelled $A$ and $B$, and administered during 12 weeks.

In total, $2 \times 189$ patients were randomized, distributed over 36 centres. Subjects were followed during 12 weeks (3 months) of treatment and followed further, up to a total of 48 
weeks (12 months). Measurements were taken at baseline, every month during treatment, and every 3 months afterwards, resulting in a maximum of 7 measurements per subject. As a first response, we consider the unaffected naillength (one of the secondary endpoints in the study), measured from the nail bed to the infected part of the nail, which is always at the free end of the nail, expressed in $\mathrm{mm}$. Obviously this response will be related to the toesize. Therefore, we will include here only those patients for which the target nail was one of the two big toenails. This reduces our sample under consideration to 146 and 148 subjects respectively. Individual profiles for 30 randomly selected subjects in each treatment group are shown in Figure 1. Our second outcome will be severity of the infection, coded as 0 (not severe) or 1 (severe). The question of interest was whether the percentage of severe infections decreased over time, and whether that evolution was different for the two treatment groups. A summary of the number of patients in the study at each time-point, and the number of patients with severe infections is given in Table 2. Note that the numbers near the end go up again, owing to intermittent missingness.

A key issue in the analysis of longitudinal data is that outcome values measured repeatedly within the same subjects tend to be correlated, and this correlation structure needs to be taken into account in the statistical analysis. This is easily seen with paired observations

\section{Treatment A}

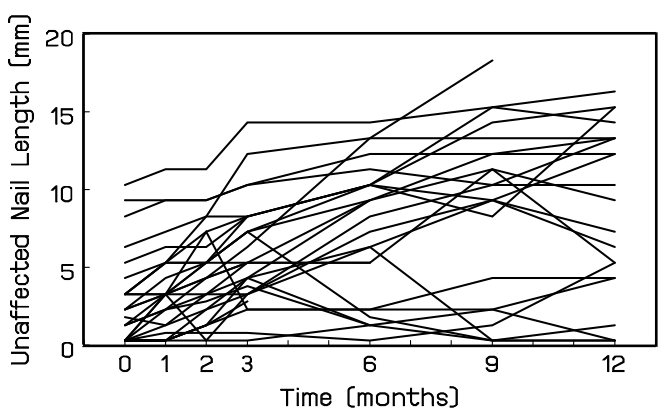

\section{Treatment B}

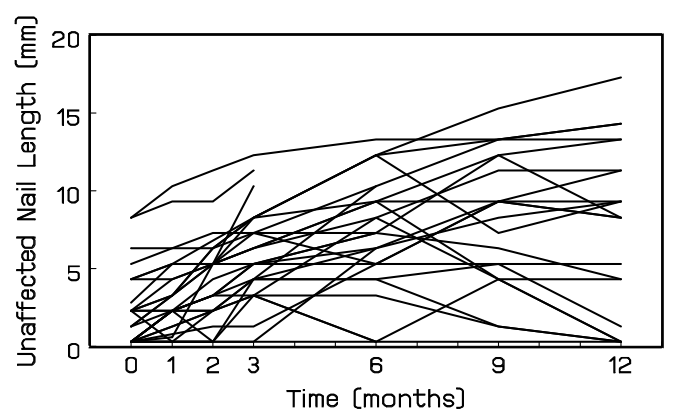

Fig. 1: Toenail data; Individual profiles of 30 randomly selected subjects in each treatment arm.

Table 2: Toenail data; Number and percentage of patients with severe toenail infection, for each treatment arm separately.

\begin{tabular}{lrrrrrr}
\hline \hline & \multicolumn{3}{c}{ Group A } & \multicolumn{3}{c}{ Group B } \\
\cline { 2 - 7 } \#aseline & 54 & 146 & $37.0 \%$ & 55 & 148 & $37.2 \%$ \\
\cline { 2 - 7 } 1 month & 49 & 141 & $34.7 \%$ & 48 & 147 & $32.6 \%$ \\
2 months & 44 & 138 & $31.9 \%$ & 40 & 145 & $27.6 \%$ \\
3 months & 29 & 132 & $22.0 \%$ & 29 & 140 & $20.7 \%$ \\
6 months & 14 & 130 & $10.8 \%$ & 8 & 133 & $6.0 \%$ \\
9 months & 10 & 117 & $8.5 \%$ & 8 & 127 & $6.3 \%$ \\
12 months & 14 & 133 & $10.5 \%$ & 6 & 131 & $4.6 \%$ \\
\hline \hline
\end{tabular}


obtained from, e.g., a pre-test/post-test experiment. An obvious choice for the analysis is the paired $t$-test, based on the subject-specific difference between the two measurements. While an unbiased estimate for the treatment effect can also be obtained from a two-sample $t$-test, standard errors and hence also $p$-values and confidence intervals obtained from not accounting for the correlation within pairs will not reflect the correct sampling variability, and hence still lead to wrong inferences. In general, classical statistical procedures assuming independent observations, cannot be used in the context of repeated measurements. In this paper, we will give an overview of the most important models useful for the analysis of clinical trial data, and widely available through commercial statistical software packages.

\subsection{Orthdontic growth data}

As an example, we use the orthodontic growth data, introduced by Potthoff and Roy (1964) and used by Jennrich and Schluchter (1986) as well. The data have the typical structure of a clinical trial and are simple yet illustrative. They contain growth measurements for 11 girls and 16 boys. For each subject, the distance from the center of the pituitary to the maxillary fissure was recorded at ages $8,10,12$, and 14 . Figure 2 presents the 27 individual profiles. Little and Rubin $(2002)$ deleted 9 of the $[(11+16) \times 4]$ measurements, rendering 9 incomplete subjects which, even though a somewhat unusual practice, has the advantage of allowing a comparison between the incomplete data methods and the analysis of the original, complete data. Deletion is confined to the age 10 measurements and roughy speaking the complete observations at age 10 are those with a higher measurement at age 8 . We will put some emphasis on ages 8 and 10, the typical dropout setting, with age 8 fully observed and age 10 partially missing.

\section{Linear models for Gaussian data}

With repeated Gaussian data, a general, and very flexible, class of parametric models is obtained from a random-effects approach. Suppose that an outcome $Y$ is observed repeatedly over time for a set of persons, and suppose that the individual trajectories are of the type as

\section{Orthodontic Growth Data \\ Profiles and Means}

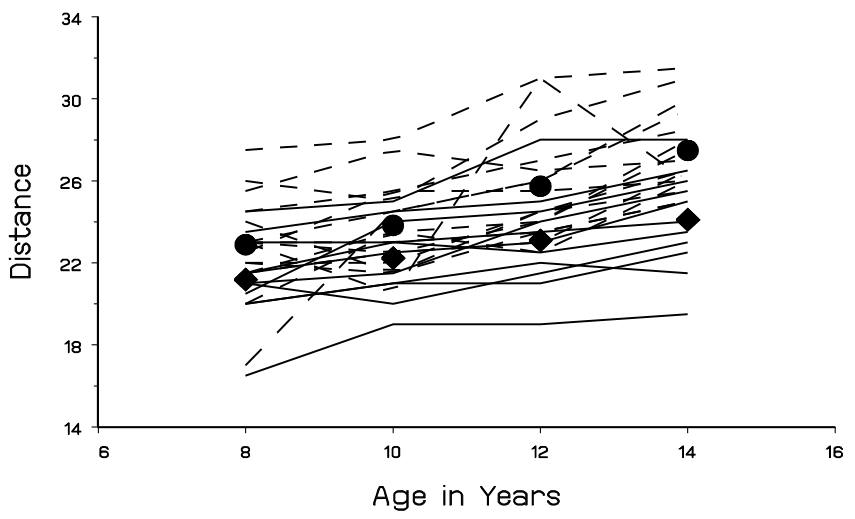

Fig. 2: Orthodontic growth data; Raw and residual profiles (Girls are indicated with solid lines; Boys are indicated with dashed lines). 


\section{Individual profiles with random intercepts and slopes}

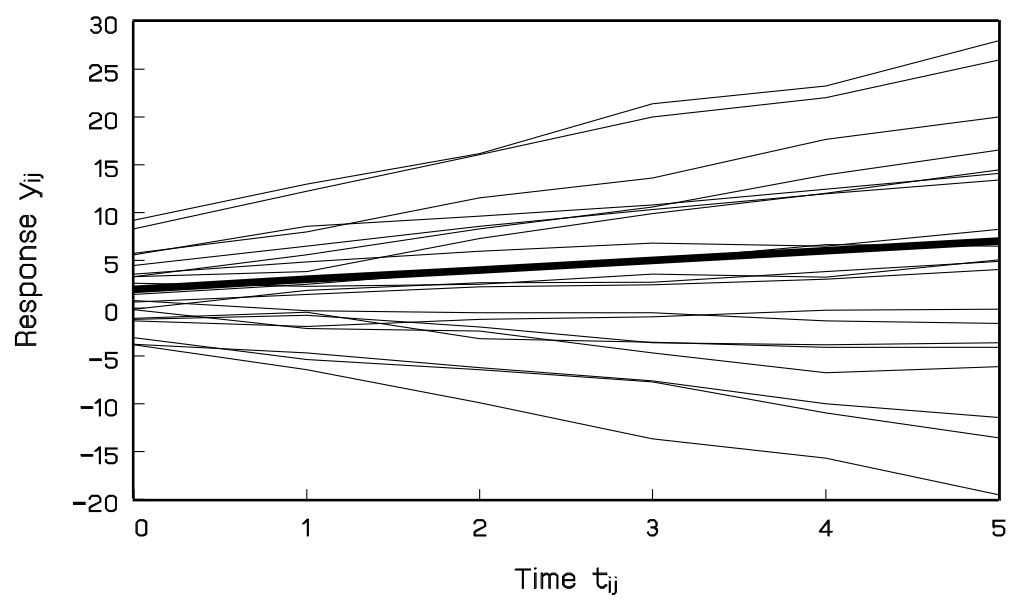

Fig. 3: Hypothetical example of continuous longitudinal data which can be well described by a linear mixed model with random intercepts and random slopes (The thin lines represent the observed subject-specific evolutions; the bold line represents the population-averaged evolution; measurements are taken at six time-points $0,1,2,3$, $4,5)$.

shown in Figure 3. Obviously, a linear regression model with intercept and linear time effect seems plausible to describe the data of each person separately. However, different persons tend to have different intercepts and different slopes. One can therefore assume that the $j$ th outcome $Y_{i j}$ of subject $i\left(i=1, \ldots, N, j=1, \ldots, n_{i}\right)$, measured at time $t_{i j}$ satisfies $Y_{i j}=\tilde{b}_{i 0}+\tilde{b}_{i 1} t_{i j}+\varepsilon_{i j}$. Assuming the vector $\tilde{\boldsymbol{b}}_{\boldsymbol{i}}=\left(\tilde{b}_{i 0}, \tilde{b}_{i 1}\right)^{\prime}$ of person-specific parameters to be bivariate normal with mean $\left(\beta_{0}, \beta_{1}\right)^{\prime}$ and $2 \times 2$ covariance matrix $D$ and assuming $\varepsilon_{i j}$ to be normal as well, this leads to a so-called linear mixed model. In practice, one will often formulate the model as

$$
Y_{i j}=\left(\beta_{0}+b_{i 0}\right)+\left(\beta_{1}+b_{i 1}\right) t_{i j}+\varepsilon_{i j},
$$

with $\tilde{b}_{i 0}=\beta_{0}+b_{i 0}$ and $\tilde{b}_{i 1}=\beta_{1}+b_{i 1}$, and the new random effects $\boldsymbol{b}_{\boldsymbol{i}}=\left(b_{i 0}, b_{i 1}\right)^{\prime}$ are now assumed to have mean zero.

The above model can be viewed as a special case of the general linear mixed model which assumes that the outcome vector $\boldsymbol{Y}_{\boldsymbol{i}}$ of all $n_{i}$ outcomes for subject $i$ satisfies

$$
\boldsymbol{Y}_{\boldsymbol{i}}=X_{i} \boldsymbol{\beta}+Z_{i} \boldsymbol{b}_{\boldsymbol{i}}+\boldsymbol{\varepsilon}_{\boldsymbol{i}},
$$

in which $\boldsymbol{\beta}$ is a vector of population-averaged regression coefficients called fixed effects, and where $\boldsymbol{b}_{\boldsymbol{i}}$ is a vector of subject-specific regression coefficients. The $\boldsymbol{b}_{\boldsymbol{i}}$ are assumed normal with mean vector 0 and covariance $D$, and they describe how the evolution of the $i$ th subject deviates from the average evolution in the population. The matrices $X_{i}$ and $Z_{i}$ are $\left(n_{i} \times p\right)$ and $\left(n_{i} \times q\right)$ matrices of known covariates. Note that $p$ and $q$ are the numbers of fixed and subject-specific regression parameters in the model, respectively. The residual components $\varepsilon_{i}$ are assumed to be independent $N\left(\mathbf{0}, \Sigma_{i}\right)$, where $\Sigma_{i}$ depends on $i$ only through its dimension $n_{i}$. Model (1) naturally follows from a so-called two-stage 
model formulation. First, a linear regression model is specified for every subject separately, modelling the outcome variable as a function of time. Afterwards, in the second stage, multivariate linear models are used to relate the subject-specific regression parameters from the first-stage model to subject characteristics such as age, gender, treatment, etc.

Estimation of the parameters in (1) is usually based on maximum likelihood (ML) or restricted maximum likelihood (REML) estimation for the marginal distribution of $\boldsymbol{Y}_{\boldsymbol{i}}$ which can easily be seen to be

$$
\boldsymbol{Y}_{\boldsymbol{i}} \sim N\left(X_{i} \boldsymbol{\beta}, Z_{i} D Z_{i}^{\prime}+\Sigma_{i}\right)
$$

Note that model (1) implies a model with very specific mean and covariance structures, which may or may not be valid, and hence need to be checked for every specific data set at hand. Note also that, when $\Sigma_{i}=\sigma^{2} I_{n_{i}}$, with $I_{n_{i}}$ equal to the identity matrix of dimension $n_{i}$, the observations of subject $i$ are independent conditionally on the random effect $\boldsymbol{b}_{\boldsymbol{i}}$. The model is therefore called the conditional independence model. Even in this simple case, the assumed random-effects structure still imposes a marginal correlation structure for the outcomes $Y_{i j}$. Indeed, even if all $\Sigma_{i}$ equal $\sigma^{2} I_{n_{i}}$, the covariance matrix in (2) is not the identity matrix, illustrating that, marginally, the repeated measurements $Y_{i j}$ of subject $i$ are not assumed to be uncorrelated. Another special case arises when the random effects are omitted from the model. In that case, the covariance matrix of $\boldsymbol{Y}_{\boldsymbol{i}}$ is modeled through the residual covariance matrix $\Sigma_{i}$. In the case of completely balanced data, i.e., when $n_{i}$ is the same for all subjects, and when the measurements are all taken at fixed time-points, one can assume all $\Sigma_{i}$ to be equal to a general unstructured covariance matrix $\Sigma$, which results in the classical multivariate regression model. Inference in the marginal model can be done using classical techniques including approximate Wald tests, $t$-tests, $F$-tests, or likelihood ratio tests. Finally, Bayesian methods can be used to obtain 'empirical Bayes estimates' for the subject-specific parameters $\boldsymbol{b}_{\boldsymbol{i}}$ in (1). We refer to Henderson et al. (1959), Harville (1974, 1976, 1977), Laird and Ware (1982), Verbeke and Molenberghs (2000) for more details about estimation and inference in linear mixed models.

As an illustration, we analyse the unaffected naillength response in the toenail example. The model proposed by Verbeke, Lesaffre and Spiessens (2001) assumes a quadratic evolution for each subject, with subject-specific intercepts, and with correlated errors within subjects. More formally, they assume that $Y_{i j}$ satisfies

$$
Y_{i j}(t)= \begin{cases}\left(\beta_{A 0}+b_{i}\right)+\beta_{A 1} t+\beta_{A 2} t^{2}+\varepsilon_{i}(t), & \text { in group A } \\ \left(\beta_{B 0}+b_{i}\right)+\beta_{B 1} t+\beta_{B 2} t^{2}+\varepsilon_{i}(t), & \text { in group B, }\end{cases}
$$

where $t=0,1,2,3,6,9,12$ is the time in the study, expressed in months. The error components $\varepsilon_{i}(t)$ are assumed to have common variance $\sigma^{2}$, with correlation of the form $\operatorname{corr}\left(\varepsilon_{i}(t), \varepsilon_{i}(t-u)\right)=\exp \left(-\varphi u^{2}\right)$ for some unknown parameter $\varphi$. Hence, the correlation between within-subject errors is a decreasing function of the time span between the corresponding measurements. Fitted average profiles are shown in Figure 4. An approximate $F$-test shows that, on average, there is no evidence for a treatment effect $(p=0.2029)$.

Note that, even when interest would only be in comparing the treatment groups after 12 months, this could still be done based on the above fitted model. The average difference between group $\mathrm{A}$ and group $\mathrm{B}$, after 12 months, is given by $\left(\beta_{A 0}-\beta_{B 0}\right)-12\left(\beta_{A 1}-\beta_{B 1}\right)+$ $12^{2}\left(\beta_{A 2}-\beta_{B 2}\right)$. The estimate for this difference equals $0.80 \mathrm{~mm}(p=0.0662)$. Alternatively, a two-sample $t$-test could be performed based on those subjects which have completed the study. This yields an estimated treatment effect of $0.77 \mathrm{~mm}(p=0.2584)$ illustrating that 


\section{Fitted averages}

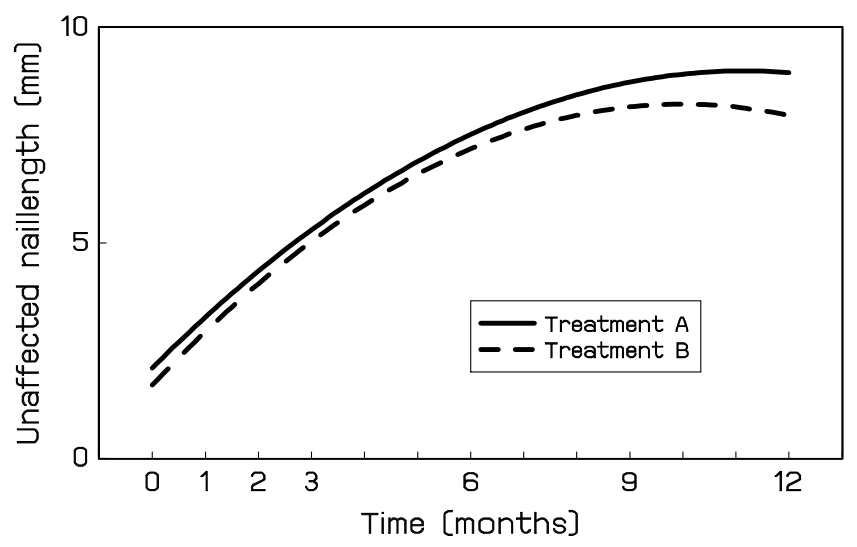

Fig. 4: Toenail data; Fitted average profiles based on model (3).

modelling the whole longitudinal sequence also provides more efficient inferences at specific time-points.

\section{Models for discrete outcomes}

Whenever discrete data are to be analysed, the normality assumption in the models in the previous section is no longer valid, and alternatives need to be considered. The classical route, in analogy to the linear model, is to specify the full joint distribution for the set of measurements $Y_{i j}, \ldots, Y_{i n_{i}}$ per individual. Clearly, this implies the need to specify all moments up to order $n_{i}$. Examples of marginal models can be found in Bahadur (1961), Altham (1978), Efron (1986), Lang and Agresti (1994), Molenberghs and Lesaffre(1994, 1999) and Fahrmeir and Tutz (2001).

Especially for longer sequences and/or in cases where observations are not taken at fixed time-points for all subjects, specifying a full likelihood, as well as making inferences about its parameters, traditionally done using maximum likelihood principles, can become very cumbersome. Therefore, inference is often based on a likelihood obtained from a randomeffects approach. Associations and all higher-order moments are then implicitly modelled through a random-effects structure. This will be discussed in Section 4.1. A disadvantage is that the assumptions about all moments are made implicitly, and are very hard to check. As a consequence, alternative methods have been in demand, which require the specification of a small number of moments only, leaving the others completely unspecified. In a large number of cases, one is primarily interested in the mean structure, whence only the first moments need to be specified. Sometimes, there is also interest in the association structure, quantified, for example using odds ratios or correlations. Estimation is then based on so-called generalized estimating equations, and inference no longer directly follows from maximum likelihood theory. This will be explained in Section 4.2. In Section 4.3, both approaches will be illustrated in the context of the toenail data. A comparison of both techniques will be presented in Section 4.4. 


\subsection{Generalized linear mixed models (GLMM)}

As discussed in Section 3, random effects can be used to generate an association structure between repeated measurements. This can be exploited to specify a full joint likelihood in the context of discrete outcomes. More specifically, conditionally on a vector $\boldsymbol{b}_{\boldsymbol{i}}$ of subjectspecific regression coefficients, it is assumed that all responses $Y_{i j}$ for a single subject $i$ are independent, satisfying a generalized linear model with mean $\mu_{i j}=h\left(\boldsymbol{x}_{\boldsymbol{i} \boldsymbol{j}} \boldsymbol{\beta}_{\boldsymbol{\beta}}+\boldsymbol{z}_{\boldsymbol{i}} \boldsymbol{j}^{\prime} \boldsymbol{b}_{\boldsymbol{i}}\right)$ for a pre-specified link function $h$, and for two vectors $\boldsymbol{x}_{\boldsymbol{i} \boldsymbol{j}}$ and $\boldsymbol{z}_{\boldsymbol{i j}}$ of known covariates belonging to subject $i$ aht the $j$ th time point. Let $f_{i j}\left(y_{i j} \mid \boldsymbol{b}_{\boldsymbol{i}}\right)$ denote the corresponding density function of $Y_{i j}$, given $\boldsymbol{b}_{\boldsymbol{i}}$. As for the linear mixed model, the random effects $\boldsymbol{b}_{\boldsymbol{i}}$ are assumed to be sampled from a normal distribution with mean vector $\mathbf{0}$ and covariance $D$. The marginal distribution of $\boldsymbol{Y}_{\boldsymbol{i}}$ is then given by

$$
f\left(\boldsymbol{y}_{\boldsymbol{i}}\right)=\int \prod_{j=1}^{n_{i}} f_{i j}\left(y_{i j} \mid \boldsymbol{b}_{\boldsymbol{i}}\right) f\left(\boldsymbol{b}_{\boldsymbol{i}}\right) d \boldsymbol{b}_{\boldsymbol{i}}
$$

in which dependence on the parameters $\boldsymbol{\beta}$ and $D$ is suppressed from the notation. Assuming independence across subjects, the likelihood can easily be obtained, and maximum likelihood estimation becomes available.

In the linear model, the integral in (4) could be worked out analytically, leading to the normal marginal model (2). In general however, this is no longer possible, and numerical approximations are needed. Broadly, we can distinguish between approximations to the integrand in (4), and methods based on numerical integration. In the first approach, Taylor series expansions to the integrand are used, simplifying the calculation of the integral. Depending on the order of expansion and the point around which one expands, slightly different procedures are obtained. We refer to Breslow and Clayton (1993) and to Wolfinger and O'Connell (1993) for an overview of estimation methods. In general, such approximations will be accurate whenever the responses $y_{i j}$ are 'sufficiently continuous' and/or if all $n_{i}$ are sufficiently large. This explains why the approximation methods perform poorly in cases with binary repeated measurements, with a relatively small number of repeated measurements available for all subjects (Wolfinger, 1998). Especially in such examples, numerical integration proves very useful. Of course, a wide toolkit of numerical integration tools, available from the optimization literature, can be applied. A general class of quadrature rules selects a set of abscissas and constructs a weighted sum of function evaluations over those. We refer to Hedeker and Gibbons $(1994,1996)$ and to Pinheiro and Bates (2000) for more details on numerical integration methods in the context of random-effects models.

\subsection{Generalized estimating equations (GEE)}

Liang and Zeger (1986) proposed so-called generalized estimating equations (GEE) which require only the correct specification of the univariate marginal distributions provided one is willing to adopt 'working' assumptions about the association structure. More specifically, a generalized linear model (McCullagh and Nelder, 1989) is assumed for each response $Y_{i j}$, modelling the mean $\mu_{i j}$ as $h\left(\boldsymbol{x}_{\boldsymbol{i j}}{ }^{\prime} \boldsymbol{\beta}\right)$ for a pre-specified link function $h$, and a vector $\boldsymbol{x}_{\boldsymbol{i}}$ of known covariates. In case of independent repeated measurements, the classical score equations for the estimation of $\boldsymbol{\beta}$ are well known to be

$$
S(\boldsymbol{\beta})=\sum_{i} \frac{\partial \boldsymbol{\mu}_{i}^{\prime}}{\partial \boldsymbol{\beta}} V_{i}^{-1}\left(\boldsymbol{Y}_{\boldsymbol{i}}-\boldsymbol{\mu}_{i}\right)=0
$$

where $\boldsymbol{\mu}_{i}=\mathrm{E}\left(\boldsymbol{Y}_{\boldsymbol{i}}\right)$ and $V_{i}$ is a diagonal matrix with $v_{i j}=\operatorname{Var}\left(Y_{i j}\right)$ on the main diagonal. Note that, in general, the mean-variance relation in generalized linear models implies that the 
elements $v_{i j}$ also depend on the regression coefficients $\boldsymbol{\beta}$. Generalized estimating equations are now obtained from allowing non-diagonal 'covariance' matrices $V_{i}$ in (5). In practice, this comes down to the specification of a 'working correlation matrix' which, together with the variances $v_{i j}$ results in a hypothesized covariance matrix $V_{i}$ for $\boldsymbol{Y}_{\boldsymbol{i}}$.

Solving $S(\boldsymbol{\beta})=0$ is done iteratively, constantly updating the working correlation matrix using moment-based estimators. Note that, in general, no maximum likelihood estimates are obtained, since the equations are not first-order derivatives of some log-likelihood function for the data under some statistical model. Still, very similar properties can be derived. More specifically, Liang and Zeger (1986) showed that $\widehat{\boldsymbol{\beta}}$ is asymptotically normally distributed, with mean $\boldsymbol{\beta}$ and with a covariance matrix that can easily be estimated in practice. Hence, classical Wald-type inferences become available. This result holds provided that the mean was correctly specified, whatever working assumptions were made about the association structure. This implies that, strictly speaking, one can fit generalized linear models to repeated measurements, ignoring the correlation structure, as long as inferences are based on the standard errors that follow from the general GEE theory. However, efficiency can be gained from using a more appropriate working correlation model (Mancl and Leroux, 1996).

The original GEE approach focuses on inferences for the first-order moments, considering the association present in the data as nuisance. Later on, extensions have been proposed which also allow inferences about higher-order moments. We refer to Prentice (1988), Lipsitz, Laird and Harrington (1991), and Liang, Zeger and Qaqish (1992) for more details on this.

\subsection{Application to the toenail data}

As an illustration of GEE and GLMM, we analyse the severity of infection binary outcome in the toenail example. We will first apply GEE, based on the marginal logistic regression model

$$
\log \left[\frac{P\left(Y_{i}(t)=1\right)}{1-P\left(Y_{i}(t)=1\right)}\right]= \begin{cases}\beta_{A 0}+\beta_{A 1} t, & \text { in group A } \\ \beta_{B 0}+\beta_{B 1} t, & \text { in group B. }\end{cases}
$$

Furthermore, we use an unstructured $7 \times 7$ working correlation matrix. The results are reported in Table 3, and the fitted average profiles are shown in the top graph of Figure 5. Based on a Wald-type test we obtain a significant difference in the average slope between the two treatment groups $(p=0.0158)$.

Alternatively, we consider a generalized linear mixed model, modelling the association through the inclusion of subject-specific (random) intercepts. More specifically, we will now

Table 3: Toenail data; Parameter estimates (standard errors) for a generalized linear mixed model (GLMM) and a marginal model (GEE).

\begin{tabular}{lrr}
\hline \hline & \multicolumn{1}{c}{ GLMM } & \multicolumn{1}{c}{ GEE } \\
\cline { 2 - 3 } Parameter & Estimate (s.e.) & Estimate (s.e.) \\
\hline Intercept group A $\left(\beta_{A 0}\right)$ & $-1.63(0.44)$ & $-0.72(0.17)$ \\
Intercept group B $\left(\beta_{B 0}\right)$ & $-1.75(0.45)$ & $-0.65(0.17)$ \\
Slope group A $\left(\beta_{A 1}\right)$ & $-0.40(0.05)$ & $-0.14(0.03)$ \\
Slope group B $\left(\beta_{B 1}\right)$ & $-0.57(0.06)$ & $-0.25(0.04)$ \\
Random intercepts s.d. $(\sigma)$ & $4.02(0.38)$ & \\
\hline \hline
\end{tabular}



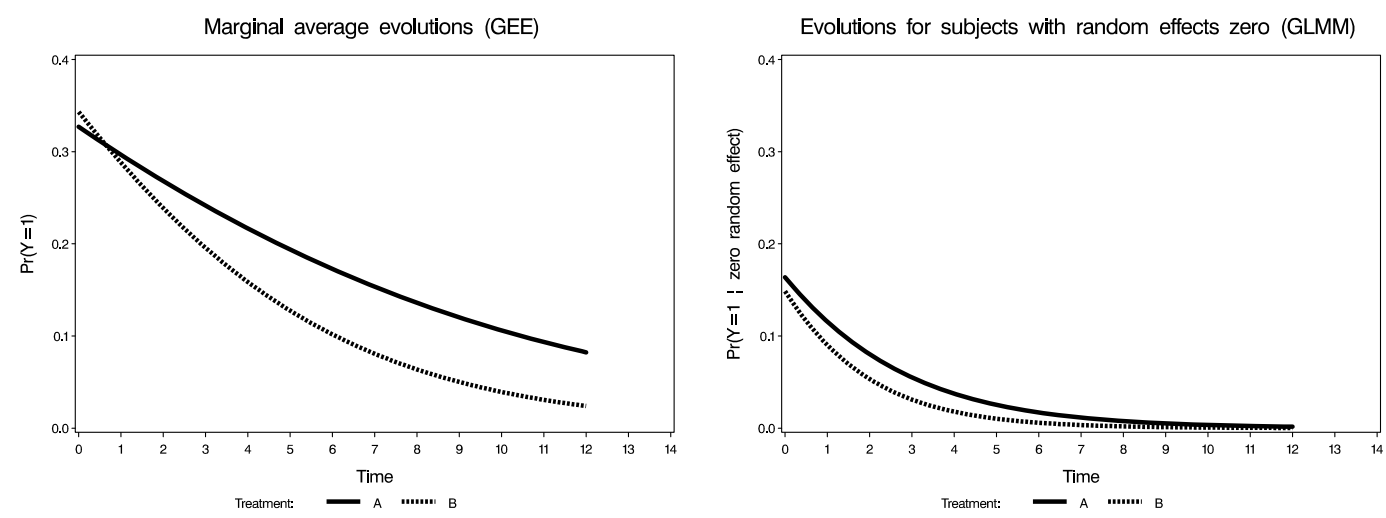

Fig. 5: Toenail data; Treatment-specific evolutions ((a) Marginal evolutions as obtained from the marginal model (6) fitted using GEE; (b) Evolutions for subjects with random effects in model (7) equal to zero).

assume that

$$
\log \left[\frac{P\left(Y_{i}(t)=1 \mid b_{i}\right)}{1-P\left(Y_{i}(t)=1 \mid b_{i}\right)}\right]= \begin{cases}\beta_{A 0}+b_{i}+\beta_{A 1} t, & \text { in group A } \\ \beta_{B 0}+b_{i}+\beta_{B 1} t, & \text { in group B }\end{cases}
$$

with $b_{i}$ normally distributed with mean 0 and variance $\sigma^{2}$. The results, obtained using numerical integration methods, are also reported in Table 3 . As before, we obtain a significant difference between $\beta_{A 1}$ and $\beta_{B 1}(p=0.0255)$.

\subsection{Marginal versus hierarchical parameter interpretation}

Comparing the GEE results and the GLMM results in Table 3, we observe large differences between the parameter estimates. This suggests that the parameters in both models need to be interpreted differently. Indeed, the GEE approach yields parameters with a population-averaged interpretation. Each regression parameter expresses the average effect of a covariate on the probability of having a severe infection. Results from the generalized linear mixed model however, require an interpretation conditionally on the random effect, i.e., conditionally on the subject. In the context of our toenail example, consider model (7) for treatment group A only. The model assumes that the probability of severe infection satisfies a logistic regression model, with the same slope for all subjects, but with subjectspecific intercepts. The population-averaged probability of severe infection is obtained from averaging these subject-specific profiles over all subjects. This is graphically presented in Figure 6. Clearly, the slope of the average trend is different from the subject-specific slopes, and this effect will be more severe as the subject-specific profiles differ more, i.e., as the random-intercepts variance $\sigma^{2}$ is larger. Formally, the average trend for group A is obtained as

$$
\begin{aligned}
P\left(Y_{i}(t)=1\right)=E\left[P\left(Y_{i}(t)=1 \mid b_{i}\right)\right] & =E\left[\frac{\exp \left(\beta_{A 0}+b_{i}+\beta_{A 1} t\right)}{1+\exp \left(\beta_{A 0}+b_{i}+\beta_{A 1} t\right)}\right] \\
& \neq E\left[\frac{\exp \left(\beta_{A 0}+\beta_{A 1} t\right)}{1+\exp \left(\beta_{A 0}+\beta_{A 1} t\right)}\right]
\end{aligned}
$$

Hence, the population-averaged evolution is not the evolution for an 'average' subject, i.e., a subject with random effect equal to zero. The right hand graph in Figure 5 shows the fitted 


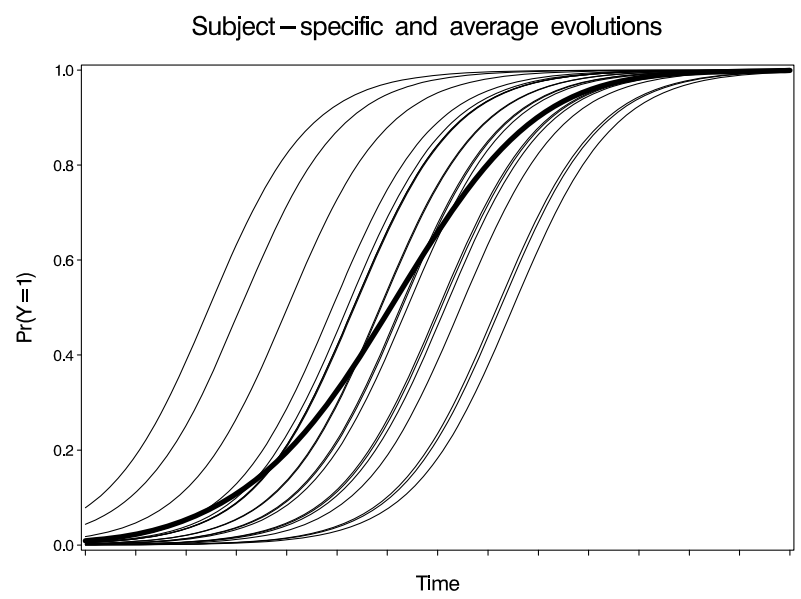

Fig. 6: Graphical representation of a random-intercepts logistic model (The thin lines represent the subject-specific logistic regression models; the bold line represents the population-averaged evolution).

profiles for an average subject in each treatment group, and these profiles are indeed very different from the population-averaged profiles shown in the left hand graph of Figure 5 and discussed before. In general, the population-averaged evolution implied by the GLMM is not of a logistic form any more, and the parameter estimates obtained from the GLMM are typically larger in absolute value than their marginal counterparts (Neuhaus, Kalbfleisch and Hauck, 1991). However, one should not refer to this phenomenon as bias since the two sets of parameters target at different scientific questions. Note that this difference in parameter interpretation between marginal and random-effects models immediately follows from the non-linear nature, and therefore is absent in the linear mixed model, discussed in Section 3. Indeed, the regression parameter vector $\boldsymbol{\beta}$ in the linear mixed model (1) is the same as the regression parameter vector modelling the expectation in the marginal model (2).

In case fully non-linear models are considered, then this framework can be generalized even further (Davidian and Giltinan, 1995; Vonesh and Chinchilli, 1997).

\section{Methods in common use for incomplete data}

Turning to the incomplete data problem, we will focus on two relatively simple methods that have been and still are in extensive use. A detailed account of simple methods to handle missingness is given in Verbeke and Molenberghs (2000).

\subsection{Complete case analysis}

A complete case analysis includes only those cases for analysis, for which all measurements were recorded. This method has obvious advantages. It is very simple to describe and since the data structure is as would have resulted from a complete experiment, standard statistical software can be used without additional work. Further, since the entire estimation is done on the same subset of completers, there is a common basis for inference. Unfortunately, the method suffers from severe drawbacks. First, there is nearly always a 
substantial loss of information. The impact on precision and power is dramatic. Further, such an analysis will only be representative for patients who remain on study. Of course a complete case analysis could have a role as an auxiliary analysis, especially if a scientific question relates to it. A final important issue about a complete case analysis is that it is only valid when the missingness mechanism is MCAR. However, severe bias can result when the missingness mechanism is MAR but not MCAR. This bias can go both ways, i.e., either overestimating or underestimating the true effect.

\subsection{Last observation carried forward}

A method that has received a lot of attention (Siddiqui and Ali, 1998; Mallinckrodt et al., 2003) is last observation carried forward (LOCF). As already noted before, in the LOCF method, whenever a value is missing, the last observed value is substituted. For the LOCF approach, the MCAR assumption is necessary but not sufficient for an unbiased estimate. Indeed, it further assumes that subjects' responses would have been constant from the last observed value to the endpoint of the trial. These conditions seldom hold (Verbeke and Molenberghs, 2000). In a clinical trial setting, one might believe that the respons profile changes as soon as a patient goes off treatment and even that it would flatten. However, the constant profile assumption is even stronger. Therefore, carrying observations forward may bias estimates of treatment effects and underestimate the associated standard errors (Heyting, Tolboom and Essers, 1992; Gibbons et al., 1993; Lavori, Dawson and Shera, 1995; Verbeke and Molenberghs, 2000; Mallinckrodt, Clark and Stacy, 2001a, b). Further, this method artificially increases the amount of information in the data, by treating imputed and actually observed values on equal footing.

Despite its shortcomings, LOCF has been the longstanding method of choice for the primary analysis in clinical trials because of its simplicity, ease of implementation, and the belief that the potential bias from carrying observations forward leads to a "conservative" analysis in comparative trials. An analysis is called conservative when it leads to no treatment difference, while in fact there is treatment difference. However, reports of anticonservative or liberal behavior of LOCF are common (Little and Yau 1996; Liu and Gould 2002; Kenward et al., 2004; Mallinckrodt et al., 2004; Molenberghs et al., 2004). This means that a LOCF analysis can create treatment effect when none exists. Thus the statement that LOCF analysis has been used to provide a conservative estimate of treatment effect is unacceptable.

Historically, an important motivation behind the simpler methods was their simplicity. Indeed, the main advantage, shared with complete case analysis, is that complete data software can be used. However, with the availability of commercial software tools, such as, for example, the SAS procedures MIXED and NLMIXED and the SPlus and R nlme libraries, this motivation no longer applies.

It is often quoted that LOCF or CC, while problematic for parameter estimation, produce randomization-valid hypothesis testing, but this is questionable. First, in a CC analysis partially observed data are selected out, with probabilities that may depend on post-randomization outcomes, thereby undermining any randomization justification. Second, if the focus is on one particular time point, e.g., the last one scheduled, then LOCF plugs in data. Such imputations, apart from artificially inflating the information content, may deviate in complicated ways from the underlying data (Kenward et al., 2004). Third, although the size of a randomization based LOCF test may reach its nominal size under the null hypothesis of no difference in treatment profiles, there will be other regions of the alternative space where the power of the LOCF test procedure is equal to its size, which is 
completely unacceptable.

\section{Alternative approaches to incomplete data}

We will first provide a graphical illustration, using an artificial example, of the various simple methods we have considered and then turn to so-called direct likelihood analysis.

\subsection{Illustration of simple methods}

Let us take a look at an artificial but insightful example, depicted in Figure 7, which displays the results of the traditional methods, CC and LOCF, next to the result of an MAR method. In this example, the mean response is supposed to be linear. For both groups (completers and dropouts), the slope is the same, but intercepts differ. Patients with incomplete observations dropped out half way of the study, e.g., because they reached a certain level of the outcome. This is obviously an MAR missingness mechanism. Using a method, valid under the MAR assumption, yields the correct mean profile, being a straight line centered between the mean profiles of the completers and incompleters. If one would perform a $\mathrm{CC}$ analysis, the fitted profile will coincide with the mean profile of the complete cases (bold line). Next, under LOCF, data are imputed (dashed line). The resulting fitted profile will be the bold dashed line. Clearly, both traditional methods produce an incorrect result.

Further, in a traditional available case analysis (AC), one makes use of the information actually available. One such set of estimators could be the treatment-specific mean at a number of designed measurement occasions. With a decreasing sample size over time, means later in time would be calculated using less subjects than means earlier in time. Figure 7 shows a dramatic instance of this, due to the rather extreme nature of this illustrative example. The key message is that such an approach is unable to remove major sources of bias.

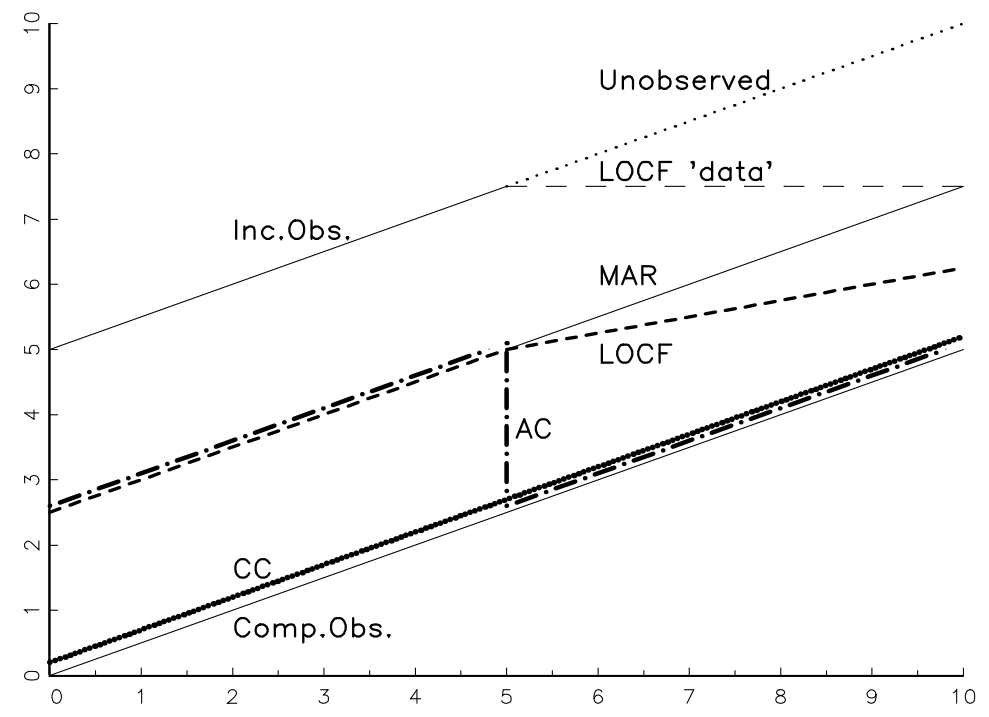

Fig. 7: Artificial situation, illustrating the results of the traditional MCAR methods, CC and LOCF, next to the result of the direct likelihood method. 


\subsection{Direct likelihood analysis}

For continuous outcomes, Verbeke and Molenberghs (2000) describe likelihood-based mixed-effects models, which are valid under the MAR assumption. Indeed, for longitudinal studies, where missing data are involved, a mixed model only requires that missing data are MAR. As opposed to the traditional techniques, mixed-effects models permit the inclusion of subjects with missing values at some time points (both dropout and intermittent missingness).

This likelihood-based MAR analysis is also termed likelihood-based ignorable analysis, or, as we will be using in the remainder of this entry, a direct likelihood analysis. In such a direct likelihood analysis, the observed data are used without deletion nor imputation. In doing so, appropriate adjustments are made to parameters at times when data are incomplete, due to the within-patient correlation.

Thus, even when interest lies, for example, in a comparison between the two treatment groups at the last occasion, such a full longitudinal analysis is a good approach, since the fitted model can be used as the basis for inference at the last occasion.

In many clinical trials, the repeated measures are balanced in the sense that a common (and often limited) set of measurement times is considered for all subjects, which allows the a priori specification of a "saturated" model. For example, a full group-by-time interaction for the fixed effects combined with an unstructured covariance matrix. The direct likelihood analysis is equivalent to a classical MANOVA analysis when data are complete, but more generally valid when they are incomplete. This is a strong answer to the common criticism that a direct likelihood method is making strong assumptions. Indeed, its coincidence with MANOVA for data without missingness shows that the assumptions made are very mild. Therefore, it constitutes a very promising alternative for CC and LOCF. When a relatively large number of measurements is made within a single subject, the full power of random effects modeling can be used (Verbeke and Molenberghs, 2000).

The practical implication is that a software module with likelihood estimation facilities and with the ability to handle incompletely observed subjects, manipulates the correct likelihood, providing valid parameter estimates and likelihood ratio values.

A few cautionary remarks are warranted. First, when at least part of the scientific interest is directed towards the nonresponse process, obviously both processes need to be considered. Under MAR, both questions can be answered separately. This implies that a conventional method can be used to study questions in terms of the outcomes of interest, such as treatment effect and time trend, whereafter a separate model can be considered to study missingness. Second, likelihood inference is often surrounded with references to the sampling distribution (e.g., to construct measures of precision for estimators and for statistical hypothesis tests; Kenward and Molenberghs, 1998). However, the practical implication is that standard errors and associated tests, when based on the observed rather than the expected information matrix and given that the parametric assumptions are correct, are valid. Thirdly, it may be hard to rule out the operation of an MNAR mechanism. This point was brought up in the introduction and will be discussed further in Section 9 .

\section{Illustration: Orthodontic growth data}

The simple methods and direct likelihood method from Sections 5 and 6 are now compared using the growth data. For this purpose, a linear mixed model is used, assuming unstructured mean, i.e., assuming a separate mean for each of the eight age $\times$ sex combinations, together with an unstructured covariance structure, and using maximum likelihood 
(ML) as well as restricted maximum likelihood (REML). The mean profiles of the linear mixed model using maximum likelihood for all four data sets, for boys, are given in Figure 8. The girls' profiles are similar and hence not shown.

Next to this longitudinal approach, we will consider a full MANOVA analysis and a univariate ANOVA analysis, i.e., one per time point. For all of these analyses, Table 4 shows the estimates and standard errors for boys at ages 8 and 10, for the original data and all available incomplete data, as well as for the CC and the LOCF data.

First, we consider the group means for the boys in the original data set in Figure 8, i.e., we observe relatively a straight line. Clearly, there seems to be a linear trend in the mean profile.

In a complete case analysis of the growth data, the 9 subjects which lack one measurement are deleted, resulting in a working data set with 18 subjects. This implies that 27 available measurements will not be used for analysis, a quite severe penalty on a relatively small data set. Observing the profiles for the CC data set in Figure 8, all group means increased relative to the original data set but mostly so at age 8 . The net effect is that the profiles overestimate the average length.

For the LOCF data set, the 9 subjects that lack a measurement at age 10 are completed by imputing the age 8 value. It is clear that this procedure will affect the apparently increasing linear trend found for the original data set. Indeed, the imputation procedure forces the means at ages 8 and 10 to be more similar, thereby destroying the linear relationship. Hence, a simple, intuitively appealing interpretation of the trends is made impossible.

In case of direct likelihood, we now see two profiles. One for the observed means and the other for the fitted means. These two coincide at all ages except age 10. As mentioned

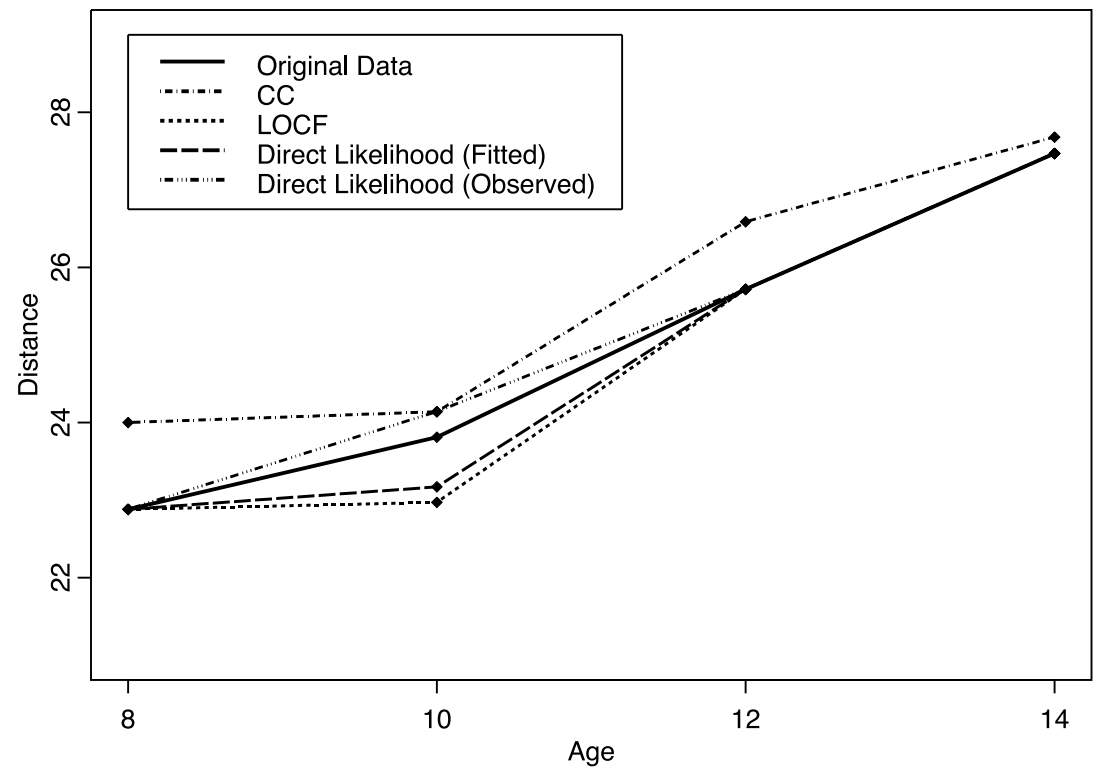

Fig. 8: Orthodontic growth data; Profiles for the original data, CC, LOCF, and direct likelihood (fitted: pertains to all planned measurements; observed: here the prediction is considered only for those measurements that were actually taken) for boys. 
Table 4: Orthodontic growth data; Comparison of analyses based on means at (completely observed age 8 and incompletely observed age 10 measurement).

\begin{tabular}{lcc}
\hline \hline Method & Boys at Age 8 & Boys at Age 10 \\
\hline \multicolumn{3}{c}{ Original Data } \\
\hline Direct likelihood, ML & $22.88(0.56)$ & $23.81(0.49)$ \\
Direct likelihood, REML & $22.88(0.58)$ & $23.81(0.51)$ \\
MANOVA & $22.88(0.58)$ & $23.81(0.51)$ \\
ANOVA per time point & $22.88(0.61)$ & $23.81(0.53)$ \\
\hline \multicolumn{3}{c}{ All Available Incomplete Data } \\
\hline Direct likelihood, ML & $22.88(0.56)$ & $23.17(0.68)$ \\
Direct likelihood, REML & $22.88(0.58)$ & $23.17(0.71)$ \\
MANOVA & $24.00(0.48)$ & $24.14(0.66)$ \\
ANOVA per time point & $22.88(0.61)$ & $24.14(0.74)$ \\
\hline \multicolumn{3}{c}{ Complete Case Analysis } \\
\hline Direct likelihood, ML & $24.00(0.45)$ & $24.14(0.62)$ \\
Direct likelihood, REML & $24.00(0.48)$ & $24.14(0.66)$ \\
MANOVA & $24.00(0.48)$ & $24.14(0.66)$ \\
ANOVA per time point & $24.00(0.51)$ & $24.14(0.74)$ \\
\hline \multicolumn{2}{c}{ Last Observation Carried Forward Analysis } \\
\hline Direct likelihood, ML & $22.88(0.56)$ & $22.97(0.65)$ \\
Direct likelihood, REML & $22.88(0.58)$ & $22.97(0.68)$ \\
MANOVA & $22.88(0.58)$ & $22.97(0.68)$ \\
ANOVA per time point & $22.88(0.61)$ & $22.97(0.72)$ \\
\hline \hline
\end{tabular}

earlier, the complete observations at age 10 are those with a higher measurement at age 8. Due to the within-subject correlation, they are the ones with a higher measurement at age 10 as well, and therefore the fitted model corrects in the appropriate direction. The consequences of this are very important. While we are inclined to believe that the fitted means do not follow the observed means all that well, this nevertheless is precisely what we should observe. Indeed, since the observed means are based on a non-random subset of the data, the fitted means take into account all observed data points, as well as information on the observed data at age 8 , through the measurements that have been taken for such children, at different time points.

As an aside to this, note that, in case of direct likelihood, the observed average at age 10 coincides with the $\mathrm{CC}$ average, while the fitted average does not coincide with anything else. Indeed, if the model specification is correct, then a direct likelihood analysis produces a consistent estimator for the average profile, as if nobody had dropped out. Of course, this effect might be blurred in relatively small data sets due to small-sample variability. Irrespective of the small-sample behavior encountered here, the validity under MAR and the ease of implementation are good arguments that favor this direct likelihood analysis over other techniques. 
Let us now compare the different methods by means of Table 4, which shows the estimates and standard errors for boys at age 8 and 10, for the original data and all available incomplete data, as well as for the CC data and the LOCF data.

Table 4 shows some interesting features. In all four cases, a CC analysis gives an upward biased estimate, for both age groups. This is obvious, since the complete observations at age 10 are those with a higher measurement at age 8 , as we have seen before. The LOCF analysis gives a correct estimate for the average outcome for boys at age 8 . This is not surprising since there were no missing observations at this age. As noted before, the estimate for boys of age 10 is biased downwards. When the incomplete data are analyzed, we see from Table 4 that direct likelihood produces good estimates. The MANOVA and ANOVA per time point analyses give an overestimation of the average of age 10, like in the $\mathrm{CC}$ analysis. Further, the MANOVA analysis also yields an overestimation of the average at age 8 , again the same as in the CC analysis.

Thus, direct likelihood shares the elegant and appealing features of ANOVA and MANOVA for fully observed data, but is superior with incompletely observed profiles.

\subsection{Generalized estimating equations and incomplete data}

Because GEE is not likelihood based; therefore ignorability (Rubin, 1976) cannot be invoked to establish the method's validity under MAR. Therefore, apart from special cases, GEE in its basic form will be valid only under MCAR. In response to this, Robins et al. (1995) proposed a class of so-called weighted estimating equations.

The idea is to weight each subject's contribution to the GEE by the inverse probability, either of being fully observed, or of being observed up to a certain time. Let $\pi_{i}$ be the probability for subject $i$ to be completely observed and $\pi_{i}^{\prime}$ the probability for subject $i$ to drop out on occasion $d_{i}$. These can be written as

$$
\pi_{i}=\prod_{\ell=2}^{n_{i}}\left(1-p_{i \ell}\right), \quad \pi_{i}^{\prime}=\left[\prod_{\ell=2}^{d_{i}-1}\left(1-p_{i \ell}\right)\right] \cdot p_{i d_{i}}
$$

where $p_{i \ell}=P\left(D_{i}=\ell \mid D_{i} \geq \ell, Y_{i \bar{\ell}}, X_{i \bar{\ell}}\right)$ are the component probabilities of dropping out at occasion $\ell$, given the subject is still in the study, the covariate history $X_{i \bar{\ell}}$ and the outcome history $Y_{i \bar{\ell}}$. In such a case, one can opt either for WGEE based on the completers only:

$$
\boldsymbol{U}(\boldsymbol{\beta})=\sum_{i=1}^{N} \frac{\widetilde{R}_{i}}{\pi_{i}} \frac{\partial \boldsymbol{\mu}_{i}}{\partial \boldsymbol{\beta}^{\prime}} V_{i}^{-1}\left(\boldsymbol{y}_{i}-\boldsymbol{\mu}_{i}\right)=\mathbf{0}
$$

with $\widetilde{R}_{i}=1$ if a subject is fully observed and 0 otherwise, or, upon using (8), for WGEE using all subjects:

$$
\boldsymbol{U}(\boldsymbol{\beta})=\sum_{i=1}^{N} \frac{1}{\pi_{i}^{\prime}} \frac{\partial \boldsymbol{\mu}_{i}^{o}}{\partial \boldsymbol{\beta}^{\prime}}\left(V_{i}^{o}\right)^{-1}\left(\boldsymbol{y}_{i}^{o}-\boldsymbol{\mu}_{i}^{o}\right)=\mathbf{0} .
$$

Here, the superscript 'o' indicates the portion corresponding to the observed data in the corresponding matrix or vector. Of course, with (9), the incomplete subjects also contribute through the model for the dropout probabilities $\pi_{i}$.

Furthermore, (9) has been extended towards so-called double robustness (Scharfstein, Rotnitzky and Robins 1999; Van der Laan and Robins 2003; Bang and Robins, 2005). 


\subsection{Multiple imputation}

A useful complement to direct likelihood and weighted generalized estimating equations is described next. Since its introduction nearly 30 years ago (Rubin, 1978) multiple imputation has become an important and influential approach for dealing with the statistical analysis of incomplete data. It now has a very large bibliography, including several reviews and texts (Rubin and Schenker, 1986; Rubin, 1987; Rubin, 1996; Schafer, 1997, 1999; Horton and Lipsitz, 2001). During this period, its domain of application has spread steadily from sample surveys to include many diverse areas such as the analysis of observational data from public health research and clinical trials. In parallel with these developments, tools for multiple imputation have been incorporated into several mainstream statistical packages. In this chapter we provide an introduction to the multiple imputation procedure and indicate some of the roles it may have to play in the current setting.

The key idea of the multiple imputation (MI) procedure is to replace each missing value with a set of $M$ plausible values. Each value is a Bayesian draw from the conditional distribution of the missing observation given the observed data, made in such a way that the set of imputations properly represents the information about the missing value that is contained in the observed data for the chosen model. The imputations produce $M$ "completed" datasets, each of which is analysed using the method that would have been appropriate had the data been complete. The model for the latter analysis is called the substantive model, while that used to produce the imputations is called the imputation model. One great strength of the MI procedure is that, to a certain extent, these two models can be considered separately. MI is most straightforward to use under MAR, and most software implementations make this assumption. However, it is quite possible to apply it in MNAR settings and this is particularly convenient when certain classes of pattern-mixture model are used to construct the imputation model. Two examples are Little and Yau (1996) and Thijs et al. (2002).

Multiple imputation involves three distinct phases or, using Rubin's (1987) terminology, tasks:

1. The missing values are filled in $M$ times to generate $M$ complete data sets.

2. The $M$ complete data sets are analyzed by using standard procedures.

3. The results from the $M$ analyses are combined into a single inference.

The actual procedure is as follows. Suppose that we are interested in making inferences about the $k \times 1$ parameter vector $\boldsymbol{\beta}$ from the substantive model and we are able to make appropriate Bayesian posterior draws from the imputation model. We return later to see how this might be done in practice. Replacing the missing data by their corresponding imputation samples, $M$ completed datasets are constructed. Denote by $\hat{\boldsymbol{\beta}}^{m}$ and $\boldsymbol{V}^{m}$ respectively the estimate of $\boldsymbol{\beta}$ and its covariance matrix from the $m$ th completed dataset, $(m=1, \ldots, M)$. The MI estimate of $\boldsymbol{\beta}$ is the simple average of the estimates:

$$
\hat{\boldsymbol{\beta}}^{*}=\frac{1}{M} \sum_{m=1}^{M} \hat{\boldsymbol{\beta}}^{m} .
$$

We also need a measure of precision for $\hat{\boldsymbol{\beta}}^{*}$ that properly reflects the uncertainty in the imputations. One great practical advantage of MI is the existence of a simple expression for the covariance matrix of $\hat{\boldsymbol{\beta}}^{*}$ that can be applied very generally and uses only complete data 
quantities. This is known as Rubin's variance formula (Rubin, 1987) and combines withinand between-imputation variability in an intuitively appealing way. Define

$$
\boldsymbol{W}=\frac{1}{M} \sum_{m=1}^{M} \boldsymbol{V}^{m}
$$

to be the average within-imputation covariance matrix, and

$$
\boldsymbol{B}=\frac{1}{M-1} \sum_{m=1}^{M}\left(\hat{\boldsymbol{\beta}}^{m}-\hat{\boldsymbol{\beta}}^{*}\right)\left(\hat{\boldsymbol{\beta}}^{m}-\hat{\boldsymbol{\beta}}^{*}\right)^{\prime}
$$

to be the between-imputation covariance matrix of $\hat{\boldsymbol{\beta}}^{m}$. Then an estimate of the covariance matrix of $\hat{\boldsymbol{\beta}}^{*}$ is given by

$$
\boldsymbol{V}=\boldsymbol{W}+\left(\frac{M+1}{M}\right) \boldsymbol{B} .
$$

Apart from an adjustment to accommodate the finite number of imputations used, this is a very straightforward combination of between- and within-imputation variability.

\section{Some reflections on computation}

Fortunately, these days, not only the simplistic methods, but also the ones advocated for practical use can be done without too much trouble. We will focus on SAS (Version 9.2) implementations, while similar considerations would hold for other packages. Various macros and additional code will be mentioned that can be down loaded from the authors' web site at www.censtat.be/software.

The only step required to perform a complete case analysis is deletion of subjects for which not all designed measurements have been obtained. When the data are organized 'horizontally,' i.e., one record per subject, this is particularly easy. With 'vertically' organized data, slightly more data manipulation is needed and the SAS macro, prepared by Caroline Beunckens, can be used. A generic form is:

\%cc (data=mydata, id=subject, time=time, response=yvar, out=outdata);

After performing this data pre-processing, a complete case analysis follows, of any type requested by the user. The macro requires records, corresponding to missing values, to be present in the dataset. Otherwise, it is assumed that a measurement occasion not included is missing by design.

Similar steps for last observation carried forward are performed using the macro:

$\%$ locf (dat a=mydata, id=subject, time=time, response=yvar, out=outdata);

When, for example, such preprocessing is to be followed by fitting a linear mixed model, a generalized linear mixed model, or generalized estimating equations, the statistician can use conventional MIXED, GLIMMIX, NLMIXED, or GENMOD code. Evidently, any other analysis can be entertained following $\mathrm{CC}$ and LOCF preprocessing. As a reminder, it is our belief that $\mathrm{CC}$ and LOCF analyses ought better not to be done. All we are doing here is describing how one would proceed, should the user be interested in such analyses.

In contrast to $\mathrm{CC}$ and LOCF, no extra data processing is necessary when a direct likelihood analysis is envisaged, provided the software tool used for analysis is able to handle 
measurement sequences of unequal length. This is the case for virtually all longitudinal data analysis tools, including the SAS procedures MIXED, GLIMMIX, NLMIXED, or GENMOD. One note of caution is relevant however. When residual correlation structures are used for which the order of the measurements within a sequence is important, such as unstructured and $\operatorname{AR}(1)$, but not simple or compound symmetry, and intermittent missingness occurs, care as to be taken to ensure that the design order within the sequence, and not the apparent order, is passed on. In the SAS procedure MIXED, a statement such as

repeated / subject=subject type=un;

is fine when every subject has, say, four designed measurements. However, when for a particular subject the second measurement is missing, there is a risk that the remaining measurements are considered the first, second, and third, rather than the first, third, and fourth. Thus, it is sensible to replace the above statement by:

repeated time / subject=subject type=un;

For the GENMOD procedure, the option 'withinsubject=time' of the REPEATED statement can be used. Note that this produces GEE and not direct likelihood. For the GLIMMIX procedure, there is no such feature. In all cases, especially when GLIMMIX is used, the proper order is passed on when a record is included, even for the missing measurements.

The NLMIXED procedure does not have a REPEATED statement, but it does have a RANDOM statement; when using it, all relevant information is contained in the actual effects that define the random effects structure. For example, the order is immaterial for a random intercepts model, and for a random slope in time, all information needed about time is passed on, for example, by the RANDOM statement:

RANDOM intercept time / subject=subject type=un;

Thus generally, with only a minimal amount of care, a direct likelihood analysis is no more complex than the corresponding analysis on a set of data that is free of missingness. The same holds for GEE based analyses when, for example, the GENMOD procedure is used.

To illustrate weighted generalized estimating equations, let us juxtapose a standard and a weighted version of a generic program. A GENMOD program for a standard GEE analysis would be:

proc genmod data=mydata;

class time treat subject;

model yvar = treat time treat*time / dist=binomial;

repeated subject=subject / withinsubject=time type=exch modelse;

run;

Let us now sketch the steps to be taken when conducting a weighted GEE analysis.

To compute the weights, one first has to fit the dropout model using, for example, logistic regression. The outcome 'dropout' is binary and indicates whether or not dropout occurs at a given time from the start of the measurement sequence until the time of dropout or the end of the sequence. Covariates in the model are the outcomes at previous occasions ('prev'), supplemented with genuine covariate information. The DROPOUT macro, constructed by Caroline Beunckens, is used to construct the variables 'dropout' and 'prev.'

Likewise, once a logistic regression has been fitted, these need to be translated into weights. These weights are defined at the individual measurement level and are equal to the 
product of the probabilities of not dropping out up to the measurement occasion. The last factor is either the probability of dropping out at that time or continuing the study. This task can be performed with the DROPWGT macro. The arguments are the same as in the DROPOUT macro, except that now also the predicted values from the logistic regression have to be passed on through the 'pred=' argument, and dropout indicator is passed on through the 'dropout=' argument.

Using these macros, the following code can be used to prepare for a WGEE analysis:

$\%$ dropout (dat a=mydata, id=subject, time=time, response=yvar, out=outdata);

proc genmod data=outdata descending;

class treat prev xvar time;

model dropout $=$ prev treat xvar time / pred dist=binomial;

ods output obstats=pred;

run;

data pred; set pred; keep observation pred; run;

data outdata; merge pred outdata; run;

$\%$ dropwgt ( dat a=outdata, id=subject, time=time, pred=pred, dropout=dropout, out=outdata2);

After these preparatory steps, we need only include the weights through the WEIGHT (or, equivalently, SCWGT) statement within the GENMOD procedure:

weight wi;

The three tasks of multiple imputation, imputation, analysis, and inference, can be conducted within SAS. Two key procedures are the MI and MIANALYZE procedures. We will briefly discuss each of the tasks in turn.

PROC MI is used to generate the imputations. It creates $M$ imputed datasets from an input dataset, physically stored in a single data set with indicator variable _IMPUTATION_ to separate the imputed copies. There is a variety of imputation mechanisms available, distinguishing between non-monotone and monotone sequences, and between continuous and categorical variables.

For imputations from a multivariate Gaussian imputation model the following MI program can be used:

proc $\mathrm{mi}$ data=mydata seed $=486048$ out=outdata simple nimpute=10 round=0.1;

var xvar yvar1 yvar2 yvar3 yvar4;

by treat;

run;

Here, it is assumed that there are four repeated measures per subject, a continuous covariate xvar and a binary treatment indicator. The option 'simple' displays simple descriptive statistics and pairwise correlations based on available cases in the input dataset. The number of imputations is specified by the option 'nimpute=,' with a default of five. The option 'round=' controls the number of decimal places in the imputed values, with no rounding by default. For example, 'round $=0.1$ ' requests a single decimal place. The 'seed=' option is used to specify a positive integer, which is used by PROC MI to start the pseudo-random number generator. The default is a value generated from the time of day from the computer's clock. Although not essential, it is useful when an analysis needs to be checked afterwards or when a seed is specified by an external source such as, for example, a regulatory authority. 
The imputation task is carried out separately for each level of the variables specified in the BY statement. For example, when there are several treatment arms, imputation can be done for each arm separately, thus not constraining the imputations in any way with respect to the relevant covariate, such as treatment assignment.

In PROC MI, one can choose between one of the three imputation mechanisms. When missingness is confined to dropout, the MONOTONE statement can, but does not have to, be used. The parametric regression method 'method=reg' as well as the non-parametric propensity score method ('method=propensity') are available. For general patterns of missingness, the MCMC statement can be used, which is the default as well. When using this option, fine-tuning can be done regarding the MCMC characteristics; for example, the default number of burn-in iterations is 200 , which can then be changed via the 'nbiter=' option. Further fine-tuning can be done as well.

In all cases, especially with MCMC, a number of options is available to flexibly control the imputation task. For example, 'ngroups=' specifies the number of groups based on propensity scores when the propensity scores method is used. For the MCMC method, one can give the initial mean and covariance estimates to start the MCMC process by the 'initial=' option. The 'pmm' option in the MCMC statement uses the predictive mean matching method to impute an observed value that is closest to the predicted value in the MCMC method. The 'regpmm' option in the MONOTONE statement uses the predictive mean matching method to impute an observed value that is closest to the predicted value for data sets with monotone missingness. One can specify more than one method in the MONOTONE statement, and for each imputed variable, the covariates can be specified separately.

Whereas such methods as 'propensity' and 'regression' are used for incomplete continuous outcomes, incomplete categorical outcomes can be imputed by including them into the CLASS statement, in addition to their inclusion in the VAR statement. In such a case, the MONOTONE option should be used, and one can make use of logistic regression and discriminant analysis imputation by means of the options 'logistic' and 'discrim,' respectively.

With the (default) 'initial=EM' option, the procedure uses the means and standard deviations from available cases as the initial estimates for the EM algorithm. The final estimates after applying the EM algorithm are then used to start the MCMC process. One can also specify 'initial=input SAS-data-set' to use a SAS dataset with the initial estimates of the mean and covariance matrix for each imputation. Further, the 'niter=' option specifies the number of iterations between imputations in a single chain, the default being 100 . Note that, after carrying out the imputation task, the data are in horizontal format and need to put in the longitudinal, or vertical, format again. We are now ready to continue with the analysis task.

The imputed datasets are then analyzed using a standard complete-data procedure. It is important to ensure that the BY statement is used to ensure that a separate analysis is carried out for each completed dataset:

by _imputation_;

Parameter estimates and their estimated covariance matrices need to be stored in appropriate output datasets, so that they can be passed on to the MIANALYZE procedure which effectively implements Rubin's combination formulae. The MIANALYZE procedure has a generic form, but some care is needed when using it because estimates and accompanying covariance matrices have different names in different SAS procedures, and the output datasets 
corresponding to these may also be organized somewhat differently. The procedure is able to handle CLASS effects as well, even though a number of columns in the corresponding output datasets are then needed to multi-index the effect.

In spite of this CLASS feature of the MIANALYZE procedure, we recommend creating appropriate indicator variables for categorical effects and interactions, as a form of defensive programming, i.e., removing one potential source of error. It also facilitates direct mapping between GEE and GLMM parameters, using, respectively, the GENMOD and NLMIXED procedures.

The GENMOD procedure can then be called for a GEE analysis:

proc genmod data=outdata3;

class time subject;

by _imputation_;

model yvar = time1 time2 time3 time4 trttime1 trttime2 trttime3 trttime4

/ noint dist=binomial covb;

repeated subject $=$ subject

/ withinsubject=time type=exch modelse;

ods output ParameterEstimates=gmparms parminfo=gmpinfo CovB=gmcovb;

run;

Apart from an otherwise irrelevant change to user-defined coding indicator variables for the categorical covariates in the model, the BY statement has been added, as well as the ODS statement, to store the parameter estimates and the covariance parameters. For the latter, the 'parminfo=' option is used next to the ' $\operatorname{covb}=$ ' option, to ensure the proper names of the covariate effects are mapped to abbreviations of type 'Prm1,' etc. Note that the 'covb=' output option works only because the 'covb' option was included into the MODEL statement. The parameter estimates are generated by default. The direct output of the GENMOD procedure will be a GEE analysis for each of the ten imputed datasets. As such, they represent an intermediate step in the full multiple imputation analysis and are of no direct scientific interest. Formal inference needs to be conducted only using the results from the inference task.

Analogously, a GLMM analysis can be conducted on the multiply imputed datasets:

proc nlmixed data=outdatat 3 qpoints $=20$ maxiter=100 technique=newrap cov ecov; by _imputation_;

eta $=$ beta $11 *$ time $1+$ beta $12 *$ time $2+$ beta $13 *$ time $3+$ beta $14 *$ time 4 +b+beta $21 *$ trttime $1+$ beta $22 *$ trttime $2+$ beta $23 *$ trttime $3+$ beta2 $4 *$ trttime 4 ;

$\mathrm{p}=\exp ($ eta) $/(1+\exp ($ eta $))$;

model bindif binary $(\mathrm{p})$;

random $\mathrm{b} \sim \operatorname{normal}(0$, tau*tau) subject=subject;

estimate 'tau2' tau*tau;

ods output ParameterEstimates=nlparms CovMatParmEst=nlcovb

AdditionalEstimates=nlparmsa CovMatAddEst=nlcovba;

run;

Apart from the BY statement, four output datasets are generated using the ODS statement. For the standard model parameters, we only need the 'parameterestimates=' and 'covmatparmest=' options. If, in addition, multiple imputation inference is requested about additional estimates, then they can be saved as well using the 'additionalestimates=' and 
'covmataddest=' options. However, it is probably better to calculate the additional estimates directly from the results of the inference task, i.e., to conduct multiple imputation inference and then calculate additional estimates, rather than the other way around. For both covariance matrices to be generated, the options 'cov' and 'ecov,' respectively, need to be included into the PROC NLMIXED statement.

Finally, PROC MIANALYZE combines the $M$ inferences into a single one, by making use of Rubin's (1987) formulae. Parameter and standard errors are passed on through a combination of the 'data=,' 'parms=,' 'covb=,' and/or 'xpxi=' options to the PROC MIANALYZE statement. Using 'data=' datasets of types COV, CORR, or EST can be passed on, as well as a dataset containing parameter estimates and standard errors. When one wants to pass on parameter estimates and variance-covariance matrices instead, it is better to use 'parms=' and 'covb=' or 'parms=' and 'xpxi=.' When the 'covb=' matrices contain generic names ('Prm1,'... ), the mapping between generic and actual parameter names is passed on using 'parminfo=.' Several options for fine tuning are also available as well in PROC MIANALYZE. For example, the within-imputation, between-imputation and total covariance matrices are printed upon including the 'wcov', 'bcov', and 'tcov' options, respectively.

The parameters or effects for which multiple imputation inference is needed are passed on by means of the MODELEFFECTS statement. Categorical effects are handled as well, after including them in the CLASS statement. As stated earlier, it is safer to create appropriate indicator variables and avoid the use of the CLASS statement, as sometimes the mapping between parameter estimates and the corresponding precision parameters is not straightforward. In principle, the MIANALYZE procedure works after applying any standard analysis, using a SAS procedure, in the analysis task, from SAS Version 9.1 onwards.

The TEST statement allows testing for hypotheses about linear combinations of the parameters. The statement is based on Rubin (1987), and uses a $t$-distribution which is the univariate version of the work by $\mathrm{Li}$, Raghunathan and Rubin (1991). See also Molenberghs and Kenward (2007).

Applying the procedure to the GEE can be done using the following code:

proc mianalyze parms=gmparms covb=gmcovb parminfo=gmpinfo wcov bcov tcov; modeleffects time1 time2 time3 time4 trttime1 trttime2 trttime3 trttime4; run;

Compared to the MI procedure, the MIANALYZE procedure is rather simple, in line with the simplicity and elegance of the pooling method. Conducting multiple imputation inference for the NLMIXED analysis is done by means of:

proc mianalyze parms=nlparms covb=nlcovb wcov bcov tcov;

modeleffects beta11 beta12 beta13 beta14 beta21 beta22 beta23 beta24; run;

\section{Sensitivity analysis}

When there is residual doubt about the plausibility of MAR, one can conduct a sensitivity analysis. While many proposals have been made, this is still a very active area of research. Obviously, a number of MNAR models can be fitted, provided one is prepared to approach formal aspects of model comparison with due caution. Such analyses can be complemented with appropriate (global and/or local) influence analyses (Verbeke et al., 2001). 
Another route is to construct pattern-mixture models, where the measurement model is considered, conditional upon the observed dropout pattern, and to compare the conclusions with those obtained from the selection model framework, where the reverse factorization is used (Michiels et al., 2002; Thijs et al., 2002). Alternative sensitivity analyses frameworks are provided by Robins, Rotnitzky and Scharfstein (1998), Forster and Smith (1998) who present a Bayesian sensitivity analysis, and Raab and Donnelly (1999). A further paradigm, useful for sensitivity analysis, are so-called shared parameter models, where common latent or random effects drive both the measurement process as well as the process governing missingness.

Nevertheless, ignorable analyses may provide reasonably stable results, even when the assumption of MAR is violated, in the sense that such analyses constrain the behavior of the unseen data to be similar to that of the observed data. A discussion of this phenomenon in the survey context has been given in Rubin, Stern and Vehovar (1995). These authors firstly argue that, in well conducted experiments (some surveys and many confirmatory clinical trials), the assumption of MAR is often to be regarded as a realistic one. Secondly, and very important for confirmatory trials, an MAR analysis can be specified a priori without additional work relative to a situation with complete data. Thirdly, while MNAR models are more general and explicitly incorporate the dropout mechanism, the inferences they produce are typically highly dependent on the untestable and often implicit assumptions built in regarding the distribution of the unobserved measurements given the observed ones. The quality of the fit to the observed data need not reflect at all the appropriateness of the implied structure governing the unobserved data. Based on these considerations, we recommend, for primary analysis purposes, the use of ignorable likelihood-based methods or appropriately modified frequentist methods. To explore the impact of deviations from the MAR assumption on the conclusions, one should ideally conduct a sensitivity analysis (Verbeke and Molenberghs, 2000).

\section{Concluding remarks}

In the Pearsonian-Fisherian era, with the advent of proper experimentation with biological systems came the headaches accompanying incomplete data. What worried our early-day colleagues most is destruction of balance or other design features. While, for example, an ANOVA with unequally-sized groups is possible, it implies a bigger challenge for the computer than its conventional, equally-sized group counterpart. Remember that, in Fisher's time, the computer was, instead of our current-day laptop, a room full of assistants. In those days, they typically were women, since Fisher was of the for us now unimaginable opinion that women should do the calculations the men did not want to do themselves. While the difference between one and seven microseconds is inconsequential, it used to be, for example, seven days for an unbalanced ANOVA rather than 'only' one day for the balanced one. Then, with a focus shift from biological and agricultural experimentation to clinical trials and observational studies in humans, supplemented with sociological, psychometric, and econometric research, the prevalence of incompleteness rose dramatically around and after World War II.

The dominant concern to restore balance and maintain design simplicity has naturally lead to such methods as complete case analysis (CC) and simple imputation methods. In other words, balance was restored by either taking bits out or by filling in gaps. It is fair to say that such methods were centerfield for roughly half a century.

Rubin's and others' work in the 1970s and 1980s, lead to a formalization of important 
missing data concepts. However, it is naive to assume that a paradigm shift took place in the late 80 s or early 90 s. Users happily stuck to $\mathrm{CC}$ and imputation methods, in particular last observation carried forward (LOCF, i.e., using the last observed value as a substitute for all subsequent, missing measurements), within a regulated clinical trial environment. Reasons for this are manifold. First, there is the usual inertia, owing in part to the complexity of, for example, the drug development process, and the existence of guidelines, rules, and regulations. LOCF has become somewhat of a fetish in certain circles. While some regulatory agencies are moving beyond this method, along with other simplistic ones, unfortunately not all seem to follow suit. Second, spreading taxonomy and concepts across users from a wide range of backgrounds and with varying levels of methodological aptitude, while desirable as such, unavoidably generates haze or even plain confusion as a side effect. The recent applied literature abounds with conceptual confusion. For example, one happily conducts simulations to compare CC, pattern-mixture models, and an ignorable analysis; this makes little sense, since these three concepts belong to three different taxonomical dimensions. First, CC belongs to the class of MCAR models, along the mechanism dimension. Second, pattern-mixture models are one of the families along the model framework dimension. Along the third dimension, ignorable and non-ignorable analyses are contrasted. Getting the concepts and their implications right in one's mind is of the essence. Third, even though good methods are available, they are of little practical value when not accompanied by proper computational devices. While the times of human computers were well behind us during the twentieth century's closing decade, and a lot of good statistical software was available indeed, this was not the case for what should already then have been standard missing data methods. The emergence of software for longitudinal data analysis made the handling of highly unbalanced data, whether or not this imbalance is provoked by missingness, routine business as of, say, the mid 90s. Even more recently, decent multiple-imputation implementations have entered the community. As a fourth and final issue slowing down a paradigm shift, the field may perceive there is a blatant lack of synthesis among theorists. Let us expand on this crucial current-day issue.

Robins et al. (1995) wrote a seminal paper on the semi-parametric analysis of incomplete data. It sparked off an important, fresh strand of research, with implications for different but related fields such as causal inference. As the Rothamsted legends have it and, more tangibly, older discussions of RSS read papers bear witness to, sharp controversy among statisticians is perennial. The $R^{2}$ issue, between the $\mathbf{R u b i n}$ and Robins schools, is but another one of these. Their among researchers in the missing-data field widely known controversies are multi-layered, including, for example, the choice between likelihood or Bayesian methods on the one hand and frequentist, semi-parametric techniques on the other hand. Controversy is to some extent beneficial, as it prevents one from lazily choosing the comfort of well trodden paths, but it also risks preventing a synthesis from emerging. Instead of digging trenches, researchers should advance methodological development that would combine the best of both schools, such as multiple imputation combined with generalized estimating equations (GEE), rather than purely likelihood-based multiple imputation, from the Rubin school, or pure weighted GEE, from the Robins side.

Syntheses like these can emerge once we acknowledge that all methods have advantages and disadvantages; this is like the usual tradeoff between bias and efficiency, which incidentally is to some extent the choice the user is facing in the choice between parametric and semi-parametric equipment. The Robins school has brought forward the beautiful theory of double robustness, giving maximal protection against model misspecification. 
The last decade has witnessed an explosion of research in the MNAR category, where missingness can depend on unobserved outcomes, in addition to information already offered by the observed ones. It is a tricky situation, since one is considering models where explanatory power is extracted from data... which one does not have. Now this is not uncommon, viz. the prevalence of random effects, latent classes, latent groups, and the like. However, such models invariably are haunted by unverifiable assumptions. This is fine when not going un-acknowledged and, after all, it ought not to be forgotten that unverifiable assumptions area already involved when assuming the missing data process is missing at random. Arguably, such models should not be used for primary analysis, but preferably find their place within a so-called sensitivity analysis. We could define the latter as an analysis in which several statistical models are considered simultaneously and/or where a statistical model is further scrutinized using specialized tools, such as diagnostic measures to detect influential observations or subjects. Sensitivity tools are actively being developed and a very preliminary synthesis can be found in Molenberghs and Kenward (2007), but we should and undoubtedly will see much more work in this area. Hopefully, methodologists will think of pairing up their creations with software tools.

In conclusion, it is time to place complete case analysis and simple imputation methods, in particular LOCF, in the Museum of Statistical Science, and consider instead ignorable likelihood analysis, ignorable Bayesian analysis, multiple imputation, and weighted estimating equations as the standard. In other words, one should enter the building at the MAR floor and not at the MCAR level. This is an assignment for statisticians in academe, industry, and the government, in particular those working for regulatory agencies around the globe. More work is needed at the MNAR level; again, not so much in search of the ultimate model that definitively overcomes the missingness problem, because such a model is statistics' Philosopher's Stone. More realistically, emphasis should be on furthering the sub-field of sensitivity analysis. We do not want to look for the ultimate model but rather we need to know what models do to our conclusions, given the availability of only a portion of the designed data. Additionally, the integration of substantive information into our modeling or sensitivity analysis exercise should be taken much more seriously than is currently the case. Learning our lesson from history, it is our duty to accompany new methodology with reliable, flexible, software implementations; this is a duty of the individual researcher and the software houses alike.

\section{Acknowledgment}

The author gratefully acknowledges support from IAP research Network P6/03 of the Belgian Government (Belgian Science Policy).

\section{REFERENCES}

Afifi, A. and Elashoff, R. (1966). Missing observations in multivariate statistics I: Review of the literature. Journal of the American Statistical Association 61, 595-604.

Altham, P.M.E. (1978). Two generalizations of the binomial distribution. Applied Statistics 27, $162-167$.

Bahadur, R.R. (1961). A representation of the joint distribution of responses to $n$ dichotomous items. In Studies in Item Analysis and Prediction, H. Solomon (Ed.), 169-176, Stanford, CA: Stanford University Press. 
Bang, H. and Robins, J.M. (2005). Doubly robust estimation in missing data and causal inference models. Biometrics 61, 962-972.

Breslow, N.E. and Clayton, D.G. (1993). Approximate inferencein generalized linear mixed models. Journal of the American Statistical Association 88, 9-25.

De Backer, M., De Keyser, P., De Vroey, C. and Lesaffre, E. (1996). A 12-week treatment for dermatophyte toe onychomycosis terbinafine $250 \mathrm{mg} /$ day vs. itraconazole $200 \mathrm{mg}$ /day: A double-blind comparative trial. British Journal of Dermatology 134, 16-17.

Dempster, A.P., Laird, N.M. and Rubin, D. B. (1977). Maximum likelihood from incomplete data via the EM algorithm (with discussion). Journal of the Royal Statistical Society, Series B 39, 1-38.

Davidian, M. and Giltinan, D.M. (1995). Nonlinear Models for Repeated Measurement Data. London: Chapman \& Hall.

Diggle, P.J., Heagerty, P., Liang, K.-Y. and Zeger, S.L.(2002). Analysis of Longitudinal Data. New York: Oxford University Press.

Efron, B. (1986). Double exponential families and their use in generalized linear regression. Journal of the American Statistical Association 81, 709-721.

Fahrmeir, L. and Tutz, G. (2001). Multivariate Statistical Modelling Based on Generalized Linear Models. Heidelberg: Springer-Verlag.

Forster, J.J. and Smith, P.W. (1998). Model-based inference for categorical survey data subject to non-ignorable non-response. Journal of the Royal Statistical Society, Series B 60, $57-70$.

Gibbons, R.D., Hedeker, D., Elkin, I., Waternaux, D., Kraemer, H.C., Greenhouse, J.B., Shea, M.T., Imber, S.D., Sotsky, S.M. and Watkins, J.T. (1993). Some conceptual and statistical issues in analysis of longitudinal psychiatric data. Archives of General Psychiatry 50, 739- 750 .

Hartley, H.O. and Hocking, R. (1971). The analysis of incomplete data. Biometrics 27, $7783-808$.

Harville, D.A. (1974) Bayesian inference for variance components using only error contrasts. Biometrika 61, 383-385.

Harville, D.A. (1976). Extension of the Gauss-Markov theorem to include the estimation of random effects. The Annals of Statistics 4, 384-395.

Harville, D.A. (1977). Maximum likelihood approaches to variance component estimation and to related problems. Journal of the American Statistical Association 72, 320-340.

Hedeker, D. and Gibbons, R.D. (1994). A random-effects ordinal regression model for multilevel analysis. Biometrics 50, 933-944.

Hedeker, D. and Gibbons, R.D. (1996) MIXOR: A computer program for mixed-effects ordinal regression analysis. Computer Methods and Programs in Biomedicine 49, 157176.

Henderson, C.R., Kempthorne, O., Searle, S.R. and on Krosig, C.N. (1959). Estimation of environmental and genetic trends from records subject to culling. Biometrics 15, 192218.

Heyting, A., Tolboom, J. and Essers, J. (1992). Statistical handling of dropouts in longitudinal clinical trials. Statistics in Medicine 11, 2043-2061.

Horton, N.J. and Lipsitz, S.R. (2001). Multiple imputation in practice: Comparison of software packages for regression models with missing variables. American Statistician 55, 244-254.

Jennrich, R.I. and Schluchter, M.D. (1986). Unbalanced repeated measures models with 
structured covariance matrices. Biometrics 42, 805-820.

Kenward, M.G., Evans, S., Carpenter, J. and Molenberghs, G. (2004). Last observation carry-forward and last observation analysis. Statistics in Medicine 23, 3241-3244.

Kenward, M.G. and Molenberghs, G. (1998). Likelihood based frequentist inference when data are missing at random. Statistical Science 12, 236-247.

Laird, N.M. and Ware, J.H. (1982). Random effects models for longitudinal data. Biometrics 38, 963-974.

Lang, J.B. and Agresti, A. (1994). Simultaneously modeling joint and marginal distributions of multivariate categorical responses. Journal of the American Statistical Association 89, 625-632.

Lavori, P.W., Dawson, R. and Shera, D. (1995). A multiple imputation strategy for clinical trials with truncation of patient data. Statistics in Medicine 14, 1913-1925.

Li, K.H., Raghunathan, T.E. and Rubin, D.B. (1991). Large-sample significance levels from multiply imputed data using moment-based statistics and an $F$ reference distributions. Journal of the American Statistical Association 86, 1065-1073.

Liang, K.-Y. and Zeger, S.L. (1986). Longitudinal data analysis using generalized linear models. Biometrika 73, 13-22.

Liang, K.-Y., Zeger, S.L. and Qaqish, B. (1992). Multivariate regression analyses for categorical data. Journal of the Royal Statistical Society, Series B 54, 3-40.

Lipsitz, S.R., Laird, N.M. and Harrington, D.P. (1991). Generalized estimating equations for correlated binary data: Using the odds ratio as a measure of association. Biometrika 78, 153-160.

Little, R.J.A. and Rubin, D.B. (2002). Statistical Analysis with Missing Data. New York: John Wiley \& Sons.

Little, R.J.A. and Yau, L. (1996). Intent-to-treat analysis in longitudinal studies with dropouts. Biometrics 52, 1324-1333.

Liu G. and Gould A.L. (2002). Comparison of alternative strategies for analysis of longitudinal trials with dropouts. Journal of Biopharmaceutical Statistics 12, 207-26.

Mallinckrodt, C.H., Clark, W.S., Carroll, R.J. and Molenberghs, G. (2003). Assessing response profiles from incomplete longitudinal clinical trial data under regulatory considerations. Journal of Biopharmaceutical Statistics 13, 179-190.

Mallinckrodt, C.H., Clark, W.S. and Stacy R.D. (2001a). Type I error rates from mixedeffects model repeated measures versus fixed effects analysis of variance with missing values imputed via last observation carried forward. Drug Information Journal 35, 12151225 .

Mallinckrodt, C.H., Clark, W.S. and Stacy R.D. (2001b). Accounting for dropout bias using mixed-effects models. Journal of Biopharmaceutical Statistics 11, 9-21.

Mallinckrodt, C.H., Watkin, J.G., Molenberghs, G. and Carroll, R.J. (2004). Choice of the primary analysis in longitudinal clinical trials. Pharmaceutical Statistics 3, 161-169.

Mancl, L.A. and Leroux, B.G. (1996). Efficiency of regression estimates for clustered data. Biometrics 52, 500-511.

McCullagh, P. and Nelder, J.A. (1989). Generalized Linear Models. London: Chapman \& Hall.

Michiels, B., Molenberghs, G., Bijnens, L., Vangeneugden, T. and Thijs, H. (2002). Selection models and pattern-mixture models to analyze longitudinal quality of life data subject to dropout. Statistics in Medicine 21, 1023-1041.

Molenberghs, G. and Kenward, M.G. (2007). Missing Data in Clinical Studies. Chichester: 
John Wiley \& Sons.

Molenberghs, G. and Lesaffre, E. (1994). Marginal modelling of correlated ordinal data using a multivariate Plackett distribution. Journal of the American Statistical Association 89, 633-644.

Molenberghs, G. and Lesaffre, E. (1999). Marginal modelling of multivariate categorical data. Statistics in Medicine 18, 2237-2255.

Molenberghs, G., Thijs, H., Jansen, I., Beunckens, C., Kenward, M.G., Mallinckrodt, C. and Carroll, R.J. (2004). Analyzing incomplete longitudinal clinical trial data. Biostatistics $\mathbf{5}, 445-464$.

Neuhaus, J.M., Kalbfleisch, J.D. and Hauck, W.W. (1991). A comparison of cluster-specific and population-averaged approaches for analyzing correlated binary data. International Statistical Review 59, 25-30.

Pinheiro, J.C. and Bates, D.M. (2000). Mixed effects models in $S$ and S-Plus. New York: Springer-Verlag.

Potthoff, R.F. and Roy, S.N. (1964). A generalized multivariate analysis of variance model useful especially for growth curve problems. Biometrika 51, 313-326.

Prentice, R.L. (1988). Correlated binary regression with covariates specific to each binary observation. Biometrics 44, 1033-1048.

Raab, G.M. and Donnelly, C.A. (1999). Information on sexual behaviour when some data are missing. Applied Statistics 48, 117-133.

Robins, J.M., Rotnitzky, A. and Scharfstein, D.O. (1998). Semiparametric regression for repeated outcomes with non-ignorable non-response. Journal of the American Statistical Association 93, 1321-1339.

Robins, J.M., Rotnitzky, A. and Zhao, L.P. (1995). Analysis of semiparametric regression models for repeated outcomes in the presence of missing data. Journal of the American Statistical Association 90, 106-121.

Rubin, D.B. (1976). Inference and missing data. Biometrika 63, 581-592.

Rubin, D.B. (1978). Multiple imputations in sample surveys: A phenomenological Bayesian approach to nonresponse. In Imputation and Editing of Faulty or Missing Survey Data, 1-23, Washington, DC: U.S. Department of Commerce.

Rubin, D.B. (1987). Multiple Imputation for Nonresponse in Surveys. New York: John Wiley $\&$ Sons.

Rubin, D.B. (1996). Multiple imputation after 18+ years. Journal of the American Statistical Association 91, 473-489.

Rubin, D.B. and Schenker, N. (1986). Multiple imputation for interval estimation from simple random samples with ignorable nonresponse. Journal of the American Statistical Association 81, 366-374.

Rubin, D.B., Stern, H.S. and Vehovar, V. (1995). Handling “don't know" survey responses: The case of the Slovenian plebiscite. Journal of the American Statistical Association 90, $822-828$.

Schafer J.L. (1997). Analysis of Incomplete Multivariate Data. London: Chapman \& Hall.

Schafer, J.L. (1999). Multiple imputation: A primer. Statistical Methods in Medical Research $8,3-15$.

Scharfstein, D.O., Rotnitzky, A. and Robins, J.M. (1999). Adjusting for nonignorable dropout using semi-parametric nonresponse models. Journal of the American Statistical Association 94, 1096-1120 (with Rejoinder, 1135-146).

Siddiqui, O. and Ali, M.W. (1998). A comparison of the random-effects pattern mixture 
model with last observation carried forward (LOCF) analysis in longitudinal clinical trials with dropouts. Journal of Biopharmaceutical Statistics 8, 545-563.

Thijs, H., Molenberghs, G., Michiels, B., Verbeke, G. and Curran, D. (2002). Strategies to fit pattern-mixture models. Biostatistics 3, 245-265.

Van der Laan, M.J. and Robins, J.M. (2003). Unified Methods for Censored Longitudinal Data and Causality. New York: Springer.

Verbeke, G., Lesaffre, E. and Spiessens, B. (2001). The practical use of different strategies to handle dropout in longitudinal studies. Drug Information Journal 35, 419-434.

Verbeke, G. and Molenberghs, G. (2000) Linear Mixed Models for Longitudinal Data. New York: Springer-Verlag.

Verbeke, G., Molenberghs, G., Thijs, H., Lesaffre, E. and Kenward, M.G. (2001). Sensitivity analysis for non-random dropout: A local influence approach. Biometrics 57, 7-14.

Vonesh, E.F. and Chinchilli, V.M. (1997). Linear and Nonlinear Models for the Analysis of Repeated Measurements. New York: Marcel Dekker Inc.

Wolfinger, R.D. (1998). Towards practical application of generalized linear mixed models. In Proceedings of the 13th International Workshop on Statistical Modeling, B. Marx and H. Friedl (Eds.), 388-395, New Orleans, Louisiana, USA.

Wolfinger, R. and O'Connell, M. (1993). Generalized linear mixed models: A pseudolikelihood approach. Journal of Statistical Computation and Simulation 48, 233-243.

(Received: August 3, 2009, Accepted: September 1, 2009) 